\title{
A model for near-wall dynamics in turbulent Rayleigh-Bénard convection
}

\author{
By S. ANANDA THEERTHAN \\ AND JAYWANT H. ARAKERI \\ Department of Mechanical Engineering, Indian Institute of Science, Bangalore-560 012, India
}

(Received 1 May 1996 and in revised form 18 May 1998)

Experiments indicate that turbulent free convection over a horizontal surface (e.g. Rayleigh-Bénard convection) consists of essentially line plumes near the walls, at least for moderately high Rayleigh numbers. Based on this evidence, we propose here a two-dimensional model for near-wall dynamics in Rayleigh-Bénard convection and in general for convection over heated horizontal surfaces. The model proposes a periodic array of steady laminar two-dimensional plumes. A plume is fed on either side by boundary layers on the wall. The results from the model are obtained in two ways. One of the methods uses the similarity solution of Rotem \& Classen (1969) for the boundary layer and the similarity solution of Fuji (1963) for the plume. We have derived expressions for mean temperature and temperature and velocity fluctuations near the wall. In the second approach, we compute the two-dimensional flow field in a two-dimensional rectangular open cavity. The number of plumes in the cavity depends on the length of the cavity. The plume spacing is determined from the critical length at which the number of plumes increases by one. The results for average plume spacing and the distribution of r.m.s. temperature and velocity fluctuations are shown to be in acceptable agreement with experimental results.

\section{Introduction}

This paper is concerned with the near-wall dynamics of turbulent free convection over horizontal surfaces. This is taken to include steady-state convection between horizontal plates kept at constant temperatures (the classical Rayleigh-Bénard convection), unsteady convection between two horizontal plates with one of the plates insulated and convection over a horizontal plate kept in a vast expanse of stationary fluid. We do not distinguish between the near-wall dynamics that occur in these various flows. This is analogous to stating that near-wall flows in turbulent pipe flow and in the turbulent boundary layer are similar.

Turbulent free convection of this type, especially Rayleigh-Bénard convection has been studied extensively (Townsend 1959; Deardorff \& Willis 1967a; Tanaka \& Miyata 1980; Adrian, Ferreira \& Boberg 1986; Castaing et al. 1989). See Siggia (1994) for a recent review. In this type of flow, large thermal gradients exist near the wall(s) while near isothermal conditions prevail away from the wall(s). Experimental evidence suggests the convective heat transfer near the wall(s) is either from thermals (intermittent release of hot fluid) or essentially line plumes (continuous release of hot fluid from a line) which move randomly. Away from the wall(s) rapid mixing leads to near-isothermal conditions. 


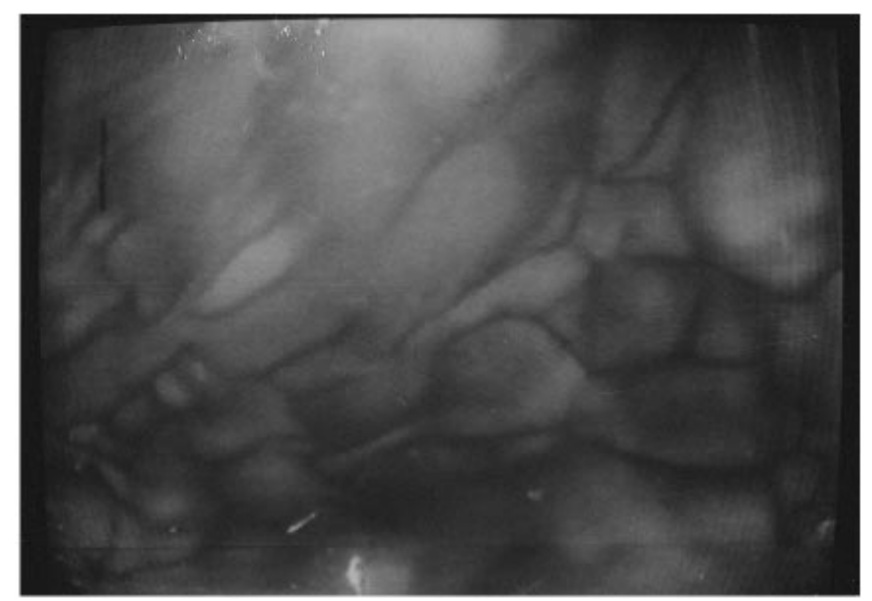

FIGURE 1. A typical planform structure obtained on a liquid crystal sheet pasted on the bottom surface of a Rayleigh-Bénard convection cell (at $R a \simeq 2 \times 10^{7}$ ). The fluid is water. The temperature difference between the bottom and top surfaces was $5{ }^{\circ} \mathrm{C}$ and the cell height was $5 \mathrm{~cm}$. The dark lines indicate higher temperature and represent sites from where plumes rise. The length of the dark line at the top left-hand corner is $D / 2$. The liquid crystal sheet is sensitive between $35^{\circ} \mathrm{C}$ and $36^{\circ} \mathrm{C}$.

Sparrow, Husar \& Goldstein (1970) observed thermals releasing from fixed sites in their experiments over a heated horizontal surface unconfined at the edges. Chu \& Goldstein (1973) also observed thermals releasing from fixed sites in a regular Rayleigh-Bénard cell at a Rayleigh number $(R a)$ of $2.23 \times 10^{6}$. They are seen as blobs getting detached from the boundary layer (figure 8 in their paper). The bulges in the boundary layer remain straight and fixed during and before the detachment.

Howard (1966) had proposed a model for intermittent release of these thermals (see for example Turner 1973). He assumed that dynamics of the flow close to the wall can be modelled as a periodic growth and eruption of a conduction layer at the wall. The conduction layer grows by diffusion, becomes unstable and erupts at a Rayleigh number, based on the conduction layer thickness, of around 1000; then a new conduction layer begins to form and the cycle repeats. The eruption of the conduction layer results in the release of a thermal. This simple model predicts average and r.m.s. temperature distributions close to the wall, the Nusselt number and the average time period between the release of thermals.

However, line plumes seem to be the predominant structure in Rayleigh-Bénard convection for $R a$ in the range $10^{6}-10^{8}$ and in convection over horizontal plates for $R a \geqslant 10^{8}$ as indicated by a large number of experiments (Chu \& Goldstein 1973; Spangenberg \& Rowland 1961; Adrian et al. 1986; Tamai \& Asaeda 1984; Theerthan \& Arakeri 1994; Goldstein \& Volino 1995). The line plumes randomly move about on the surface, inclined forward in the direction of motion. Adjacent plumes merge with one another most of the time and rarely disappear (Theerthan \& Arakeri 1994). Rayleigh-Bénard convection (at $R a \simeq 2 \times 10^{7}$ ), as visualized by a liquid crystal sheet on the bottom surface is shown in figure 1 . The cause for the movement of the plumes and how the velocity of this movement scales are still unclear. Asaeda \& Watanabe (1989) report unsteady temperature signals from a probe placed vertically above a line plume. They ascribe the unsteadiness to thermals released from the plume. However, flapping of the plume could cause similar unsteadiness.

Recent studies, with water as the working fluid, by Zocchi, Moses \& Libchaber (1990) at high $R a \sim 10^{9}$, in a cell of aspect ratio $(A R)$ one and Gluckman, Willaime \& 
Gollub (1993) at $R a$ in the range of $10^{7}$ to $3 \times 10^{8}$, in cells of aspect ratio close to one and less than one, reveal different types of structures. They used encapsulated liquid crystal particles which change colour with temperature to visualize the thermal field and described the life cycle of large events at fairly large Rayleigh numbers. Erupting warm plumes break off from the lower boundary layer and traverse the cell to the upper boundary layer where they excite waves. The waves can lead to the formation of cold plumes which then descend to the lower boundary layer, where they again excite waves. These authors describe the existence of 'spiralling swirls' or mushroom types of structures, which are basically regions of the thermal boundary layer that have been folded into a spiral. Zocchi et al. (1990) report that at $R a=2.4 \times 10^{8}$ spiralling swirls are not observed but only plumes. The existence of large-scale flow (that is, spanning the the entire cell width and height) as reported in these two papers seems to make the flow field different from the observations in large- $A R$ cells.

In the Rayleigh-Bénard convection literature two 'classes' of Nusselt number $(\mathrm{Nu})$ Rayleigh number $(R a)$ correlations have been proposed. One is from Globe \& Dropkin (1959)

$$
N u=0.069 \operatorname{Ra}^{1 / 3} \operatorname{Pr}^{0.074}
$$

(The quantities have the usual definitions: $N u=h D / k_{f}, R a=g \beta\left(2 T_{w}\right) D^{3} / v \alpha, P r=$ $v / \alpha$, where $h$ is the convective heat transfer coefficient, $D$ is the distance between the plates, $2 T_{w}$ is the difference between bottom surface temperature and top surface temperature, $k_{f}$ is the thermal conductivity, $g$ is the acceleration due to gravity, $\alpha$ is the thermal diffusivity, $\beta$ is the volume coefficient of thermal expansion and $v$ is the kinematic viscosity of the fluid.) This correlation implies that heat flux is independent of distance between the plates. The other correlation based on recent experimental data (Chu \& Goldstein 1973; Castaing et al. 1989; Wu \& Libchaber 1992) covering a wider range of Rayleigh numbers $\left(10^{4}-10^{9}\right)$,

$$
N u=C R a^{2 / 7},
$$

implies that heat flux reduces with increase in distance between the plates. $C$ depends on aspect ratio of the cell and the Prandtl number. It is of interest to note that Wu \& Libchaber (1992) found that $C=0.146$ for $A R=6.7$ and $C=0.22$ for $A R=1.0$ but the exponent was the same for both the aspect ratios. Also in the $A R=6.7$ case the above correlation was valid for $R a>10^{4}$ (much before the soft-to-hard turbulence transition at $R a \simeq 10^{8}$ (Wu \& Libchaber 1992)).

\subsection{Convection over a horizontal plate}

Another common configuration, though less extensively studied than RayleighBénard convection, is convection over a large heated horizontal plate. Two papers are of interest to us. One is by Husar \& Sparrow (1968) who visualized the convection in water over different planform shapes and covering a range of Rayleigh numbers. The other is the recent paper by Kitamura \& Kimura (1995) who measured heat transfer rates and visualized the surface temperature using liquid crystal sheets on rectangular plates. At large enough $R a$ the flow near the surface consists of (i) a laminar boundary layer near the edge followed by (ii) longitudinal rolls perpendicular to the edges and (iii) in the central region a flow structure very similar to what is observed in turbulent Rayleigh-Bénard convection: randomly moving line plumes. In region (iii) the local heat flux is observed to be independent of distance from the leading edge.

Visualizations from the side were reported by others (Rotem \& Classen 1969; Fuji \& Imura 1972; Pera \& Gebhart 1973a,b). They reported that laminar boundary layers 
exist near the plate edges and then break down or separate when the value of $G r_{x}^{1 / 3}$ exceeds about $80\left(G r_{x}\right.$ is the Grashoff number based on distance from the leading edge). Rotem \& Classen (1969) integrated the governing equations for laminar flow using similarity methods and visualized experiments in air using a semi-focusing schlieren apparatus.

The $N u-R a$ correlation for convection over a horizontal plate is $N u_{h p}=0.12 R a_{h p}^{1 / 3}$ (Kitamura \& Kimura 1995) in region (iii). Here $N u_{h p}$ and $R a_{h p}$ are based on the difference between plate temperature and ambient temperature as temperature scale; the subscript $h p$ is used to denote horizontal plate. Since $N u \sim R a^{1 / 3}$, the heat flux does not depend on the length scale.

The similarity of the flows near the wall in Rayleigh-Bénard convection and in convection over a horizontal surface is further strengthened by nearly the same heat fluxes in the two cases. Using the correlation of Globe \& Dropkin (1959) for Rayleigh-Bénard convection and that of Kitamura \& Kimura (1995) for convection over a horizontal surface we obtain the heat flux

$$
q=C T_{w}^{4 / 3} k_{f}\left(\frac{g \beta}{\alpha v}\right)^{1 / 3}
$$

with $C=0.157$ for Rayleigh-Bénard convection and 0.12 for convection over horizontal surface. Note that $T_{w}$ is the difference in temperature between the wall and the ambient or core. (As discussed above experiments in Rayleigh-Bénard convection show the exponent to be closer to $2 / 7$ than $1 / 3$. In any case, the heat flux calculation for the Rayleigh numbers of interest does not change much.)

\subsection{Outer flow}

Similar to the wall-bounded turbulent forced flows we have two scales in turbulent convection: the inner or wall scale $\left(U_{w}, Z_{w}\right)$ and the outer scale $\left(U_{o}, Z_{*}\right)$. These are shown schematically for the two flows we are considering in figure 2 . Till now we have been discussing the near-wall flow, which is the main focus of the present paper. A relevant question, raised by the recent high Rayleigh number experiments in helium (Castaing et al. 1989), is how are the two flows coupled. In particular how does the 'wind' $\left(U_{o}\right)$ affect the near-wall flow.

In Rayleigh-Bénard convection the outer velocity scale is the Deardorff (Deardorff 1970) scale denoted by $W_{*} ; W_{*}$ increases in relation to the inner velocity scale as $R a$ increases: $W_{*} / U_{w} \sim R a^{n / 3}$ (where $n$ is the exponent in the $N u-R a$ correlation) (see $\S 1.3$ ). As, for example, the distance between the plates is increased keeping the other quantities $\left(v, \alpha, 2 T_{w}\right)$ constant, the outer velocity increases consistent with the fact that fluid has a larger vertical distance over which it can gain velocity due to buoyancy. Thus at some high enough $R a$ the outer flow will start to affect the dynamics near the wall (and is believed to be related to the transition to 'hard turbulence'). This $R a$ appears to be of the order of $10^{8}$ (Wu \& Libchaber 1992). Indeed, at very high $R a$, when $W_{*} \gg U_{w}$, only the outer flow will be buoyancy driven and flow near the wall will be one of 'forced' convection (see Siggia 1994) with temperature being just a passive variable.

The effect of the outer flow is still unresolved. One hypothesis, motivated by $n$ being less than $1 / 3$, is that the outer flow stabilizes the boundary layer leading to lower heat flux with increased $R a$ (Castaing et al. 1989). Experiments and numerical simulation of natural convection flows with superimposed shear (convection on inclined plates, for example) show formation of 'longitudinal rolls'. Whether the longitudinal rolls 
(a)

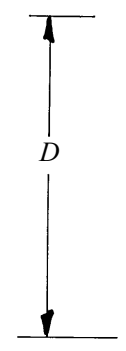

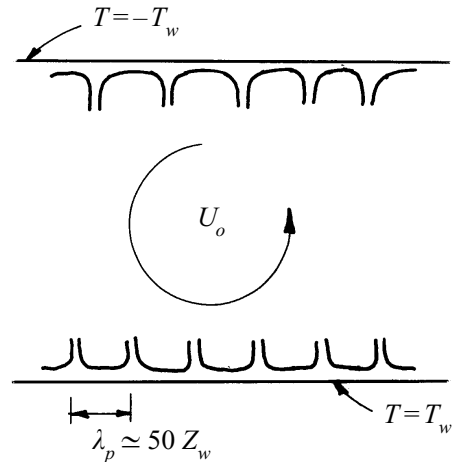

(b)

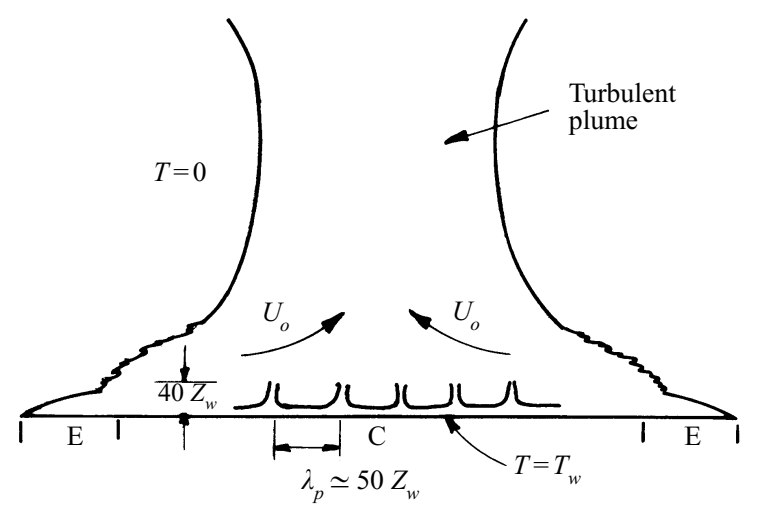

FIGURE 2. (a) Schematic of Rayleigh-Bénard convection showing plume structure near the walls. We find the average plume spacing is about $50 Z_{w}$ and height of the plume region is about $40 Z_{w}$. The outer flow eddy is shown having a velocity $U_{o}$ which scales with $W_{*}$. (b) Natural convection over a heated horizontal plate. Region $\mathrm{E}$ is the edge affected zone which contains a laminar boundary layer and longitudinal rolls. In the central region $C$, near the surface, the plumes structure is same as in $(a)$. The outer flow with velocity $U_{o}$ is due to the turbulent plume.

exist in high Rayleigh number convection is not clear; at least they are not observed in flow visualization experiments of Zocchi et al. (1990).

For convection over a horizontal plate from flow visualization the outer flow appears to be a 'turbulent' boundary layer (to our knowledge no velocity measurements have been made for such flows) (Kitamura \& Kimura 1995; Fuji \& Imura 1972). However, no outer velocity scale similar to the Deardorff scale has been proposed for this configuration. It is of interest to note here that Kitamura \& Kimura (1995) have observed the line plumes to become aligned parallel to the longer dimension in convection over the $1500 \times 500 \mathrm{~mm}$ plate. They have used $250 \mathrm{~mm}$ high fences along the longer sides in their experiments. The fences seem to 'direct' the outer flow which in turn causes the observed alignment of the plumes.

\subsection{Scaling}

The two scaling variables - inner and outer - in Rayleigh-Bénard convection were introduced by Townsend (1959) and Deardorff (1970).

Townsend's scales pertain to the conduction layer close to the boundaries. In the conduction layer, diffusion is important and the relevant physical variables are $\alpha, v$, 
$(\beta g)$, and $Q_{o}$, the kinematic heat flux $\left(=q / \rho C_{p}\right)$ respectively. Dimensional analysis gives Townsend's scales for velocity, temperature and length:

$$
W_{o}=\left(g \beta Q_{o} \alpha\right)^{1 / 4}, \quad \theta_{o}=\frac{Q_{o}}{W_{o}}, \quad Z_{o}=\frac{\alpha}{W_{o}} .
$$

Alternatively with $T_{w}$ as the independent variable instead of $Q_{o}$ we get the following inner scales for velocity, temperature and length:

$$
U_{w}=\left(g \beta T_{w}\right)^{1 / 3}(v \alpha)^{1 / 6}, \quad \theta_{w}=T_{w}, \quad Z_{w}=\frac{(\alpha v)^{1 / 2}}{U_{w}} .
$$

Equal 'weight' has been given almost arbitrarily to $v$ and $\alpha$. Clearly each of the terms may be multiplied by any power of Prandtl number. It would be desirable, if possible, to incorporate the correct Prandtl number dependence in each of the scales. This point will be discussed later in the paper. We use $U_{w}, \theta_{w}$ and $Z_{w}$ or Townsend's scales to normalize the results.

Far from the boundaries, in the interior, heat is transferred mainly by convection, in the presence of a small temperature gradient. This region occupies most of the distance between the plates. Here the relevant physical variables are $Q_{o},(\beta g)$ and $Z_{*}$ $\left(Z_{*}=D\right.$ in Rayleigh-Bénard convection). The scales for velocity, temperature and length, first introduced by Deardorff (1970) are

$$
W_{*}=\left(\beta g Q_{o} Z_{*}\right)^{1 / 3}, \quad \theta_{*}=\frac{Q_{o}}{W_{*}}, \quad Z_{*} .
$$

As this layer is considered inviscid in the absence of any kind of diffusion, a balance of kinetic energy and the potential energy would give the above set of scales.

It is useful to list the relations between the various sets of scales:

$$
Z_{*} / Z_{o}=(\operatorname{RaNuPr})^{1 / 4}, \quad \theta_{*} / \theta_{o}=(\operatorname{RaNuPr})^{-1 / 12}, \quad W_{*} / W_{o}=(\operatorname{RaNuPr})^{1 / 12} .
$$

Furthermore,

$$
W_{*}=\frac{\alpha}{Z_{*}}(\operatorname{RaPrNu})^{1 / 3}, \quad \frac{\theta_{*}}{\Delta T}=\left(\frac{N u^{2}}{\operatorname{RaPr}}\right)^{1 / 3} .
$$

The relationship between the two wall layer scales can be written as

$$
Z_{w} / Z_{o}=\left(\frac{R a}{N u^{3} \operatorname{Pr}^{3} 16}\right)^{-1 / 12}, \quad 2 \theta_{w} / \theta_{o}=\left(\frac{R a P r}{N u^{3}}\right)^{1 / 4}, \quad U_{w} / W_{o}=\left(\frac{R a P r^{3}}{N u^{3} 16}\right)^{1 / 12} .
$$

\subsection{Models for turbulent flows based on coherent structures}

No satisfactory theory or model has been proposed for the near-wall flow structure in turbulent convection over a heated surface. Howard's theory is for intermittent eruption of thermals which, however, is not often observed in experiments. Various power laws, based on mixing length arguments, have been proposed for the variation with distance in the vicinity of the wall of mean temperature and temperature fluctuations (Kraichnan 1962; Malkus 1954). These theories, however, have not found experimental verification (see for example Somerscales \& Gazda 1969). The aim of this paper is to propose a model for the near-wall structure and calculate distributions of mean temperature, r.m.s. fluctuations of temperature and of the horizontal and vertical components of velocity. The region we are considering is the conduction layer and the plume region (what is called the mixing zone by Castaing et al. 1989). The basic idea is that we model the turbulent flow near the wall as an array of 'coherent structures'. The coherent structure we recognize from experiments is the line plume. 
We first briefly review similiar models which have been suggested for other turbulent flows. The largest number is perhaps for the so-called sublayer streaks or sublayer vortices observed in wall-bounded turbulent flows (turbulent boundary layer, turbulent pipe flow, etc.). Coles (1978) modelled the flow as a periodic array of steady streamwise vortices (secondary flow) superposed on a base flow. He assumed the spacing of the vortices to be 100 wall units which is the experimentally observed mean value. The amplitude of the secondary motion was determined to fit measured profiles near the wall of mean velocity and velocity fluctuation.

More sophisticated models for the sublayer flow have been proposed to explain the dynamics (bursting and sweep phenomena) and the structure of the flow. These include the models based on proper orthogonal decomposition (POD) (Lumley 1967; Aubry et al. 1988; Sanghi \& Aubry 1993) and on linearized Navier-Stokes equations (e.g. Landahl 1990). All of them look at only the sublayer with the interaction with the outer flow modelled in some way. An interesting variant has been the minimal flow unit (Jiménez \& Moin 1991) - the smallest unit of flow that can be turbulent and also represent the important processes.

Models have also been proposed for the fine scales of isotropic turbulence in an effort to recover the Kolmogorov $k^{-5 / 3}$ scaling for the energy spectrum and to understand the mechanism of balance between vorticity production through straining and dissipation of viscosity (see Townsend 1951; Lundgren 1982; Pullin \& Saffman 1993 and references therein). Many of them are vortex based models using spatial ensembles of small-scale structures represented by local solutions of the Navier-Stokes equations. Lundgren (1982) showed that an ensemble of slender nearly axisymmetric spiral vortices embedded in a strain field predicts the Kolmogorov scaling. Pullin \& Saffman (1993) calculate higher-order one-point velocity derivative statistics using this model and assuming an ensemble of randomly oriented vortices.

Besides making simplifying assumptions, models for both the sublayer and the fine scales of isotropic turbulence depend on experimental or DNS data to make quantitative predictions. For example, Coles (1978) uses the experimentally observed value of 100 wall units for spacing between the vortices and in the LundgrenTownsend model used by Pullin \& Saffman (1993) parameter values are chosen to give correct predicted values of Kolmogorov prefactor and skewness. It is desirable a model make quantitative predictions, be simple and contain the essential physics. Its value increases if it is based on local solutions of the Navier-Stokes equations, because then, at least locally, the balance of various forces is exact. It is noteworthy that these models have been proposed for the small scales with the effect of the flow due to large scales incorporated in some way. In a sense the 'solutions' at the smaller scales are laminar and thus are amenable to such modelling, i.e., as ensemble of structures.

\subsection{Present formulation}

Coming back to the flow we are modelling, i.e. near-wall flow in turbulent free convection over a horizontal surface, the questions (as in the sublayer) are:

what is(are) the basic coherent structure(s)?

what is their role?

how are they maintained?

and regarding the model itself the questions are:

what are the assumptions?

what are the inputs to the model?

As mentioned above we assume the coherent structure to be a line plume. In the real 
(a)

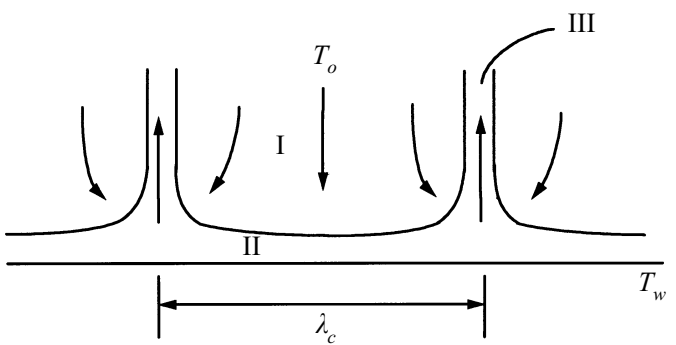

(b)

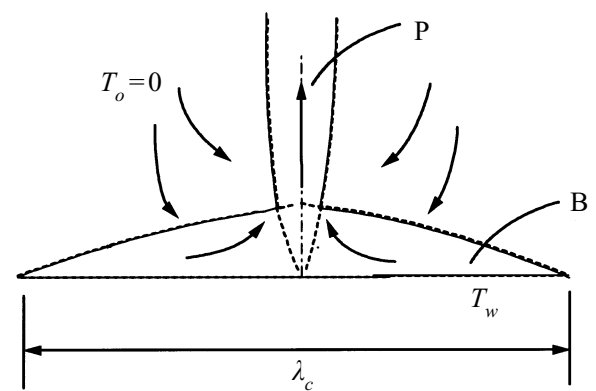

(c)

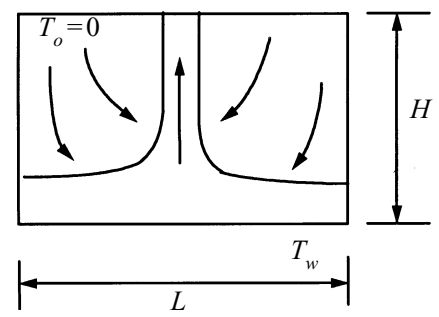

Figure 3. (a) The present model: a periodic array of two-dimensional plumes. $(b)$ The solid line is the approximation of $(a)$ that is solved using the similarity solution for plume $(\mathrm{P})$ and laminar boundary layer (B) (see text for an explanation). The similarity solutions are shown as dotted lines. (c) Computational domain and the flow structure when one plume is present.

flow the plumes are randomly spaced about some average value. They presumably have a distribution of strengths and they move about (on the horizontal surface). We model the flow by a periodic array of two-dimensional steady laminar plumes with a spacing of $\lambda_{c}$ (see figure $3 a$ ); assumptions and limitations of the model are discussed below. The flow consists of three regions: (I) Inflow of core fluid with temperature $T_{o}$, (II) boundary layer flow and (III) a plume with fluid being fed by the boundary layers on either side. The point at which the boundary layer becomes a plume may be expected to occur when the boundary layer becomes unstable.

We calculate the flow field using two methods. In the first method we combine the similarity solution of Rotem \& Classen (1969) for the boundary layer (region II) and the similarity solution of Fuji (1963) for the plume (region III) (figure $3 b$ ). Note that Rotem \& Classen's (1969) solution is for a boundary layer starting from an edge and thus figure $3(b)$ is not a true representation of the model given in figure $3(a)$. In the second method we compute the flow field in a two-dimensional cavity of height $H$ and length $L$ (figure $3 c$ ). The bottom wall is at constant temperature $T_{w}$ and satisfies the no-slip condition. The sidewalls are adiabatic and allow slip. The 
computation is carried out for two fluids: water and air. The numerical simulation is an exact solution of the model whereas the first method gives an approximate solution.

The main input to the model is the plume spacing $\left(\lambda_{c}\right)$. In the two methods we use to calculate the flow the plume spacing is obtained in two different ways. In the method based on similarity solutions we calculate the plume spacing by equating the heat flux obtained from the model to the heat flux given by a standard $\mathrm{Nu}$ $R a$ correlation. The numerical simulation gives an important result that there is a maximum distance between plumes. An increase in spacing between two plumes greater than this maximum value gives rise to a third plume. We choose the plume spacing $\lambda_{c}$ equal to half the maximum distance.

From the flow field we calculate the heat transfer rate and profiles of mean temperature and r.m.s. of temperature fluctuations and of the horizontal and vertical components of velocity. The means are calculated by averaging over one wavelength. The mean, denoted by angular brackets \langle\rangle , of any variable $\phi$ is

$$
\langle\phi\rangle=\frac{1}{\lambda_{c}} \int_{0}^{\lambda_{c}} \phi(X, Z) \mathrm{d} X ;
$$

the r.m.s. of the fluctuation, $\phi^{\prime}(X, Z)=\phi-\langle\phi\rangle$ is given by

$$
\left\langle\phi^{\prime 2}\right\rangle^{1 / 2}=\left(\frac{1}{\lambda_{c}} \int_{0}^{\lambda_{c}} \phi^{\prime 2} \mathrm{~d} X\right)^{1 / 2} .
$$

In an experiment usually a fixed probe is used and temporal averages are taken. The variation in the measured quantity with time will be due to the plumes randomly moving about. In a random field an average over several simultaneous measurements in a horizontal plane (spatial average) will also give the same value as the temporal average from a fixed probe. In the real flow the plumes will be randomly oriented on the horizontal surface. Equations (1) and (2) are the spatial averages for the two-dimensional array of plumes of the model. The assumption of the model is that flow field associated with the plume in the two-dimensional array is same as that associated with the plume in the real flow. In the model, of course, the unsteady flow due to the lateral movement of the plumes is not accounted for.

A number of assumptions have been made in the model. The assumption of laminar flow is justified in that we are dealing with near-wall flow where viscous effects are important. The Reynolds number based on the conduction layer thickness and the maximum of r.m.s. of horizontal velocity fluctuations (which occurs close to the heated wall) is around 10. The justification for using a two-dimensional model for a three-dimensional flow is that since the basic element is a line plume the flow at least locally is two-dimensional. Besides, visualizations show that often neighbouring plumes are nearly parallel. Perhaps the most serious shortcoming of the model is not incorporating the lateral motion of the plumes. However, a rational approach is not possible in the absence of a clear understanding of the cause of the motion. As we shall see the results show that the omission is perhaps not that serious.

Another assumption (which as argued above, is valid for moderate Rayleigh numbers) is that the outer flow does not influence the flow near the wall. A similar debate has been going on in the case of turbulent boundary layers. For example, is the bursting phenomenon in the sublayer influenced or caused by the outer flow? Only the recent analysis of DNS data (Brooke \& Hanratty 1993; Bernard, Thomas $\&$ Handler 1993) seems to have somewhat resolved the issue. 
In the model we assume the plume spacing to be a single (non-dimensional) value. The model can be extended to include a distribution of plume spacings. The effect of plume spacing on the results is briefly discussed in $\S 4$.

The paper is organized as follows. The calculation of the flow based on similarity solutions is given in $\$ 2$. Numerical simulation of the model is given in $\S 3$. The results along with comparison with experimental results are presented in $\$ 4$ followed by discussion in $\S 5$. Details of the calculation based on the similarity solutions are given in Appendices at the end of the paper.

\section{Calculations from similarity solutions}

The flow field in the boundary layer (region II) is calculated using the similarity solution of Rotem \& Classen (1969) and in the plume (region III) using the similarity solution of Fuji (1963); flow in region I is obtained from continuity as shown in this section. The hot plate is at a temperature $T_{w}$ and the core fluid is at a temperature $T_{o}$ which without loss of generality is taken equal to 0 . The critical length at which the boundary layer turns into a plume is $\lambda_{c} / 2$. The amount of heat required for the plume solution is equated to the heat gained in the two boundary layers by the fluid. The virtual origin for the plume calculated using the mass flux in the boundary layers comes very close to the horizontal boundary. Details of the derivations for the boundary layer are given in Appendix A and for the plume in Appendix B.

\subsection{Boundary layer}

Rotem \& Classen (1969) have presented solutions in terms of the similarity variable

$$
\eta=\left(\frac{Z}{\lambda_{c}}\right) R a_{\lambda_{c}}^{1 / 5} \operatorname{Pr}^{-1 / 5}\left(\frac{X}{\lambda_{c}}\right)^{-2 / 5},
$$

where the horizontal length scale is chosen as $\lambda_{c} / 2$ in the present paper. Here $X$ is in the horizontal direction and $Z$ is in the vertically upward direction. $G r_{c}=$ $g \beta\left(\lambda_{c} / 2\right)^{3} T_{w} / v^{2}$. The Rayleigh number based on the length scale $\lambda_{c}, R a_{\lambda_{c}}$, is then equal to $8 G r_{c} P r$. The thermal boundary layer thickness, $\Delta$, is given by,

$$
\Delta / \lambda_{c}=\eta_{\Delta} \operatorname{Ra}_{\lambda_{c}}^{-1 / 5} \operatorname{Pr}^{1 / 5}\left(X / \lambda_{c}\right)^{2 / 5}
$$

where $\eta_{\Delta}$ is the point where the non-dimensional temperature $\theta=T / T w=0.02$ (Rotem \& Classen 1969 define boundary layer thickness this way and we retain it).

The value of the similarity variable $\eta$ at $X=\lambda_{c} / 2$ is

$$
\eta_{c}=2^{2 / 5}\left(\frac{Z}{\lambda_{c}}\right) R a_{\lambda_{c}}{ }^{1 / 5} \mathrm{Pr}^{-1 / 5}
$$

Let $\Delta_{c}$ be the thermal boundary layer thickness at $X=\lambda_{c} / 2$. Hence, using equations (3) and (4), we find

$$
R a_{\lambda_{c}}=8 R a_{\Delta_{c}}^{5 / 2} \operatorname{Pr}^{-3 / 2} \eta_{\Delta}^{-15 / 2} ;
$$

(6) gives the relation between plume spacing and the critical boundary layer thickness $\left(\Delta_{c}\right)$. The model requires the value of $R a_{\lambda_{c}}$ to be specified for subsequent calculation. This is done in $\$ 2.5$.

The mean of any quantity is obtained by taking the spatial average over one period or wavelength (equation (1)). In terms of the similarity variable the mean of 
a variable $\phi$ is

$$
\langle\phi\rangle=5\left(\frac{Z}{\lambda_{c}}\right)^{5 / 2} \operatorname{Ra}_{\lambda_{c}}^{1 / 2} \operatorname{Pr}^{-1 / 2} \int_{\eta_{c}}^{\infty} \eta^{-7 / 2} \phi(\eta) \mathrm{d} \eta .
$$

Knowing temperature and velocity from Rotem \& Classen (1969) and using (7) we can derive expressions for distributions in the boundary layer of mean and r.m.s. temperature fluctuations and r.m.s. of horizontal velocity fluctuations. Details of the derivation are given in Appendix A and the final expressions are given in §2.4.

\subsection{Heat flux}

The average heat flux is given by

$$
\langle q\rangle=-\frac{5 \times 2^{2 / 5}}{3} k_{f}(g \beta)^{1 / 3} v^{-2 / 3} T_{w}^{4 / 3} \operatorname{Ra}_{\lambda_{c}}^{-2 / 15} \operatorname{Pr}^{2 / 15} H^{\prime}(0) .
$$

The amount of heat $\left(\mathrm{W} \mathrm{m}^{-1}\right)$ that feeds the plume is $\langle q\rangle \lambda_{c}$.

The Nusselt number for Rayleigh-Bénard convection is given by

$$
N u=\frac{\langle q\rangle D}{2 T_{w} k_{f}}=-\frac{5 \times 2^{1 / 15}}{6} H^{\prime}(0) R a_{\lambda_{c}}^{-2 / 15} \mathrm{Pr}^{-1 / 5} \mathrm{Ra}^{1 / 3}
$$

where, as mentioned earlier, $R a=g \beta 2 T_{w} D^{3} / v \alpha$ is the Rayleigh number based on $D$, the distance between the plates, and the temperature difference between the plates $2 T_{w}$. In convection over a horizontal surface with temperature difference taken as that between the plate and ambient fluid $N u$ will be the right-hand side of (9) multiplied by $2^{4 / 3}$.

\subsection{Plume}

The flow field in region III (see figure 3 ) is calculated using the similarity solutions of Fuji (1963) for a laminar line plume. The flow in the inflow region (region I) is calculated from continuity. The mass flux at any horizontal cross-section $Z$ (includes regions I and III) is zero. Thus we calculate the velocity in region I satisfying this condition and by assuming that the velocity is vertical and uniform. The heat flux required for the plume is obtained from the boundary layer solution (equation (8)). Details of the derivations to arrive at expressions for distributions of the various flow quantities in regions I and III are given in Appendix B.

Fuji (1963) gives expressions for vertical velocity $W$ and temperature $T$ in terms of the similarity variable $\xi$ (see Appendix B). The velocity in the inflow region (region $\mathrm{I})=W_{d}$ is given by the zero mass flux condition:

$$
W_{d}\left(\lambda_{c} / 2-\delta_{p} / 2\right)=\int_{0}^{\delta_{p} / 2} W \mathrm{~d} X .
$$

The expression for $\left\langle W^{2}\right\rangle$ after taking account of contributions from the plume and the inflow region becomes

$$
\left\langle W^{2}\right\rangle=\frac{1}{\lambda_{c} / 2} \int_{0}^{\delta_{p} / 2} W^{2} \mathrm{~d} X+\frac{\left(\lambda_{c}-\delta_{p}\right)}{\lambda_{c}} W_{d}^{2} .
$$

We have assumed that only the vertical component of velocity is present in region I and that it is uniform. Numerical simulation (\$3) of the flow in the cavity shows that this assumption is reasonable. The distributions of mean temperature and r.m.s. of the temperature fluctuations are calculated in a similar way. Temperature in region III is given by Fuji's (1963) solution; in region I it is zero. 
The plume thickness $\delta_{p}$ required in the above expression is

$$
\frac{\delta_{p}}{\lambda_{c}}=\xi_{c}\left[-10 / 3 H^{\prime}(0)\right]^{-1 / 5}\left(R a_{\lambda_{c}} / 8\right)^{-6 / 25} \operatorname{Pr}^{11 / 25}\left(\frac{Z}{\lambda_{c} / 2}\right)^{2 / 5} .
$$

\subsection{Expressions}

The final expressions for the distributions of the various quantities are given below. The velocity, length and temperature scales for the near-wall flow are

$$
\begin{aligned}
U_{w} & =\left(g \beta T_{w}\right)^{1 / 3}(v \alpha)^{1 / 6}, \\
Z_{w} & =\left(\frac{v \alpha}{g \beta T_{w}}\right)^{1 / 3}, \\
\theta_{w} & =T_{w} .
\end{aligned}
$$

The expressions for the velocity and temperature (see Appendices) in the boundary layer region (I) and the region above it are scaled using the above set of scales.

The distributions of the various quantities in the boundary layer are given by

$$
\begin{gathered}
\frac{\langle T\rangle_{b}}{T_{w}}=5\left(\frac{Z}{Z_{w}}\right)^{5 / 2}\left[\operatorname{Ra}_{\lambda_{c}}^{-1 / 3} \operatorname{Pr}^{-1 / 2}\right] \int_{\eta_{c}}^{\infty} H(\eta) \eta^{-7 / 2} \mathrm{~d} \eta \\
\frac{\left\langle T^{\prime 2}\right\rangle_{b}}{T_{w}^{2}}=5\left(\frac{Z}{Z_{w}}\right)^{5 / 2}\left[R a_{\lambda_{c}}^{-1 / 3} \operatorname{Pr}^{-1 / 2}\right] \int_{\eta_{c}}^{\infty} H^{2}(\eta) \eta^{-7 / 2} \mathrm{~d} \eta \\
-25\left(\frac{Z}{Z_{w}}\right)^{5}\left[\operatorname{Ra}_{\lambda_{c}}^{-2 / 3} \operatorname{Pr}^{-1}\right]\left[\int_{\eta_{c}}^{\infty} H(\eta) \eta^{-7 / 2} \mathrm{~d} \eta\right]^{2}, \\
\frac{\left\langle U^{\prime 2}\right\rangle_{b}^{1 / 2}}{U_{w}}=\sqrt{5}\left(\frac{Z}{Z_{w}}\right)^{7 / 4}\left[R a_{\lambda_{c}}^{-1 / 6} \operatorname{Pr}^{-1 / 4}\right]\left(\int_{\eta_{c}}^{\infty} \eta^{-9 / 2} F^{\prime 2}(\eta) \mathrm{d} \eta\right)^{1 / 2},
\end{gathered}
$$

and in the region above the boundary layer, by

$$
\begin{aligned}
& \frac{\langle T\rangle_{p}}{T_{w}}=\left(\frac{Z}{Z_{w}}\right)^{-1 / 5}\left[\left(R a_{\lambda_{c}} / 8\right)^{-16 / 75} \operatorname{Pr}^{-24 / 75}\right]\left[-10 / 3 H^{\prime}(0)\right]^{3 / 5} \int_{0}^{\xi_{c}} h(\xi) \mathrm{d} \xi, \\
& \frac{\left\langle T^{\prime 2}\right\rangle_{p}}{T_{w}^{2}}=\left(\frac{Z}{Z_{w}}\right)^{-4 / 5}\left[\left(R a_{\lambda_{c}} / 8\right)^{-4 / 75} \operatorname{Pr}^{-6 / 75}\right] \operatorname{Pr}^{-1}\left[-10 / 3 H^{\prime}(0)\right]^{7 / 5} \int_{0}^{\xi_{c}} h^{2}(\xi) \mathrm{d} \xi \\
&-\left(\frac{Z}{Z_{w}}\right)^{-2 / 5}\left[\left(R a_{\lambda_{c}} / 8\right)^{-32 / 75} \operatorname{Pr}^{-48 / 75}\right]\left[-10 / 3 H^{\prime}(0)\right]^{6 / 5}\left[\int_{0}^{\xi_{c}} h(\xi) \mathrm{d} \xi\right]^{2}, \\
& \frac{\left\langle W^{\prime 2}\right\rangle_{p}}{U_{w}^{2}}=\left\{\left[-10 / 3 H^{\prime}(0)\right]^{3 / 5}\left(\frac{Z}{Z_{w}}\right)^{4 / 5}\left[\left(R a_{\lambda_{c}} / 8\right)^{-16 / 75} \operatorname{Pr}^{-24 / 75}\right] \int_{0}^{\xi_{c}} f^{\prime 2}(\xi) \mathrm{d} \xi\right. \\
&+\frac{1}{1-\left(\frac{1}{2} \delta_{p} / Z_{w}\left(R a_{\lambda_{c}} / 8\right)^{-1 / 3}\right)} \\
&\left.\times\left(\frac{Z}{Z_{w}}\right)^{6 / 5}\left[-10 / 3 H^{\prime}(0)\right]^{2 / 5} \operatorname{Pr}\left[\left(R a_{\lambda_{c}} / 8\right)^{-44 / 75} \operatorname{Pr}^{9 / 75}\right]\left[\int_{0}^{\xi_{c}} f^{\prime}(\xi) \mathrm{d} \xi\right]^{2}\right\} .
\end{aligned}
$$

Note that in equations (16) to (18) the $Z$ dependence of the various quantities is not 
obvious as the lower limit, $\eta_{c}$, in the integrals is a function of $Z$ (see equation (5)). The terms $H(\eta), F^{\prime}(\eta)$ can be evaluated using the results given in Rotem \& Classen (1969) and $h(\xi), f^{\prime}(\xi)$ from those given in Fuji (1963). In general they are functions of $P r$.

The non-dimensional heat transfer, $H^{\prime}(0)$, occurring in these expressions can be written as

$$
-H^{\prime}(0)=0.3826 \operatorname{Pr}^{0.2692} .
$$

The only input now required for calculation of the profiles of temperature etc. is the plume spacing or equivalently $R a_{\lambda_{c}}$. Evaluation of $R a_{\lambda_{c}}$ is done in the next section.

\subsection{Evaluation of plume spacing}

We estimate the value of $R a_{\lambda_{c}}$ by making use of the correlation for the Nusselt number, $N u=0.069 \mathrm{Ra}^{1 / 3} \mathrm{Pr}^{0.074}$ from Globe \& Dropkin (1959) and equate it to equation (9). Then we obtain

$$
\begin{aligned}
R a_{\lambda_{c}}^{1 / 3} & =52 P r^{-0.012} \\
& =51, \quad \operatorname{Pr}=6.0, \\
& =52, \quad \operatorname{Pr}=0.7 .
\end{aligned}
$$

Equation (23) implies that $R a_{\lambda_{c}}$ is only weakly dependent on $P r$ and is approximately $1.4 \times 10^{5} ;(23)$ is an important result of the present work. The flux Rayleigh number based on plume spacing can be written using (8) and (22) as

$$
R a_{\lambda_{c_{f}}}=R a_{\lambda_{c}} \frac{\langle q\rangle \lambda_{c}}{k_{f} T_{w}}=0.84 R a_{\lambda_{c}}^{6 / 5} \operatorname{Pr}^{0.0692} .
$$

It is of interest to calculate the value $R a_{\Delta_{c}}$, the critical Rayleigh number at which the boundary layer becomes unstable and turns upwards. On the assumption that the plume is a result of an instability we may expect $R a_{\Delta_{c}} \simeq 1000$, the critical Rayleigh number value for fixed-free boundary conditions. We find, however, using equation (6),

$$
\begin{aligned}
R a_{\Delta_{c}} & =5789 \operatorname{Pr}^{-0.38}, \\
& =2932, \quad P r=6.0, \\
& =6634, \quad P r=0.7 .
\end{aligned}
$$

The difference may be due to the fact that the critical Rayleigh number of 1000 is for a linear temperature profile in still fluid whereas we have a nonlinear temperature profile and a non-zero flow velocity.

For convection on a horizontal plate the boundary layer 'separates' at some distance from the leading edge. We may expect the separation distance to be of the same order as the plume spacing calculated above. It may, however, be noted that since aligned plumes are observed just before the separation occurs the two situations are not the same. Rotem \& Classen (1969) obtained the cube root of the critical Rayleigh number based on the separation length to be around 100. Pera \& Gebhart $(1973 a, b)$, through experiments in air, found this value to be 80 while the stability calculations for $\operatorname{Pr}=0.7$ show a higher value of 122 . Recent experimental work by Kitamura \& Kimura (1995) in water show this value to be 63. All these values are higher than but of the same order as that given in (27). 


\subsection{Relation with Townsend's scaling}

For later reference we now give the relations between the wall scales, $Z_{w}, T_{w}, U_{w}$, we are using and the wall scales, $Z_{o}, \theta_{o}, W_{o}$, proposed by Townsend (1959).

Using the definition of kinematic flux, $Q_{o}$, and the expression for $\langle q\rangle$ (equation (8)), we obtain

$$
\frac{\langle q\rangle}{\rho C_{p}}=Q_{o}=(\alpha g \beta)^{1 / 3} T_{w}^{4 / 3} \frac{5 \times 2^{2 / 5}}{3} H^{\prime}(0) R a_{\lambda_{c}}^{-2 / 15} \operatorname{Pr}^{-8 / 15} .
$$

Then the relations between the two wall layer scales are

$$
\begin{gathered}
Z_{w}=Z_{o}\left[-5 / 3 H^{\prime}(0)\right]^{1 / 4}\left(\operatorname{Ra}_{\lambda_{c}} / 8\right)^{-1 / 30} \operatorname{Pr}^{1 / 5}, \\
T_{w}=\theta_{o}\left(R a_{\lambda_{c}} / 8\right)^{1 / 10} \operatorname{Pr}^{2 / 5}\left[-5 / 3 H^{\prime}(0)\right]^{-3 / 4}
\end{gathered}
$$

and

$$
U_{w}=W_{o}\left(R a_{\lambda_{c}} / 8\right)^{1 / 30} \operatorname{Pr}^{9 / 30}\left[-5 / 3 H^{\prime}(0)\right]^{-1 / 4} .
$$

The expressions (16) to (21) for distributions of the various quantities may be rewritten in terms of Townsend's variables using the above relations.

\section{Numerical solution}

In the procedure to calculate the flow using the similarity solutions there are a number of approximations: (i) the boundary layer starts with zero thickness at a leading edge (figure $3 b$ ) whereas it will be closer to a stagnation-flow boundary layer at the start in the real flow we are trying to model; (ii) we have patched three flows, namely boundary layer, plume and the downward irrotational flow (region I) which may not be strictly valid as the Reynolds numbers are not large enough; (iii) we obtain the critical plume spacing using the $N u-R a$ correlation. Of course, the main advantage of the method is that we obtain analytical expressions for the flow field. The dependence of the various variables on vertical distance and the Prandtl number is easily seen.

The numerical solution presented in this section answers two questions. One, how valid are the approximations listed above? Two, does there exist a critical plume spacing and what is its value?

We solve for one period of a periodic array of plumes as shown in figure $3 c$. The steady two-dimensional Navier-Stokes equations for an incompressible fluid, with the Boussinesq approximation are

$$
\begin{aligned}
\frac{\partial U}{\partial X}+\frac{\partial W}{\partial Z} & =0 \\
{\left[U \frac{\partial U}{\partial X}+W \frac{\partial U}{\partial Z}\right] / P r } & =-\frac{\partial p}{\partial X}+\left[\frac{\partial^{2} U}{\partial X^{2}}+\frac{\partial^{2} U}{\partial Z^{2}}\right] \\
{\left[U \frac{\partial W}{\partial X}+W \frac{\partial W}{\partial Z}\right] / P r } & =-\frac{\partial p}{\partial Z}+\left[\frac{\partial^{2} W}{\partial X^{2}}+\frac{\partial^{2} W}{\partial Z^{2}}\right]+R a T \\
U \frac{\partial T}{\partial X}+W \frac{\partial T}{\partial Z} & =\left[\frac{\partial^{2} T}{\partial X^{2}}+\frac{\partial^{2} T}{\partial Z^{2}}\right]
\end{aligned}
$$

Here $(X, Z)$ and $(U, W)$ represent the (horizontal, vertical) components of the nondimensional coordinate and the velocity, respectively. For our problem, the velocity 
is normalized by $\alpha / L$ and temperature by $T_{w} . L$ is the length of the computational domain (figure $3 c$ ).

\subsection{Boundary conditions}

The boundary conditions correspond to no-slip on the bottom wall, and symmetry or reflective conditions on the sidewalls.

The conditions imposed on the bottom and side boundaries are

$$
\begin{aligned}
& W(X, 0)=U(X, 0)=0 \quad \text { (no slip), } \\
& T(X, 0)=1.0, \\
& U(0, Z)=U(L, Z)=0 \quad \text { (no crossflow), } \\
& \frac{\partial W}{\partial X}=0 \quad \text { at } \quad X=0 \quad \text { and } \quad X=L, \\
& \frac{\partial T}{\partial X}=0 \quad \text { at } \quad X=0 \quad \text { and } \quad X=L,
\end{aligned}
$$

At the top open wall we assume

$$
\begin{aligned}
& \frac{\partial W}{\partial Z}(X, H)=U(X, H)=0 \\
& \frac{\partial T}{\partial Z} \quad \text { at } \quad(X, H)=0 \quad \text { if } W \text { is +ve and } T(X, H)=0 \text { otherwise. }
\end{aligned}
$$

That is, we assume that the temperature is equal to zero for the fluid coming into the domain and the temperature gradient is zero for the fluid leaving the domain.

We solve the above system of equations on uniform grids, using Patankar's (1980) algorithm modified for pressure to take into account the top zero normal derivative condition (Van Doormaal \& Raithby 1984) (SIMPLEC algorithm). The numerical code was validated with the results of De Vahl Davies (1983). The grid dependence was checked by carrying out the computations for the highest $G r$ encountered in grids of sizes $61 \times 61,81 \times 81$ and $121 \times 121$. The results changed by less than $1 \%$ when the grid size was increased from $81 \times 81$ to $121 \times 121$. We have retained the solutions for $121 \times 121$ grid.

The solution procedure is as follows. We compute for several values of $G r_{L}$ $\left(=g \beta L^{3} T_{w} / v^{2}\right)$. The values of $L$ are around the value of plume spacing obtained in equation (29) for $\operatorname{Pr}=0.7$ and equation (28) for $\operatorname{Pr}=6.0$. Depending on the value of $L$ either a half plume or a full plume is obtained in the solution domain (the half-plume and one plume flow structures are schematically shown in figure 5). At some particular value of $G r_{L}$ the flow changes from one-plume to a half-plume. The numerical solution confirms the hypothesis on which our model is based: there is some maximum distance between adjacent plumes. Figure 4 shows a typical single plume structure for $\mathrm{Pr}=6.0$.

The height, $H$, of the computational domain was chosen so that asymptotic distributions of various quantities like average temperature were obtained at the top of the domain. As far as the near-wall dynamics of turbulent convection is concerned flow only to a height of about half the plume spacing is likely to be relevant.

Figure 5 shows $N u / L$, average heat flux, versus $\mathrm{Gr}_{L}^{1 / 3}$ for $\operatorname{Pr}=6.0$. At transition, when the solution switches from half-plume to one-plume, the $N u / L$ value jumps. Note that $N u / L$ values at $G r_{L}^{1 / 3}=15.36$ and 30.72 are same as is to be expected as the flow structure is exactly the same. For $G r_{L}^{1 / 3}<28$ half a plume is obtained in the domain and for $G r_{L}^{1 / 3}>29$ one plume is obtained. We choose the domain length 
(a)

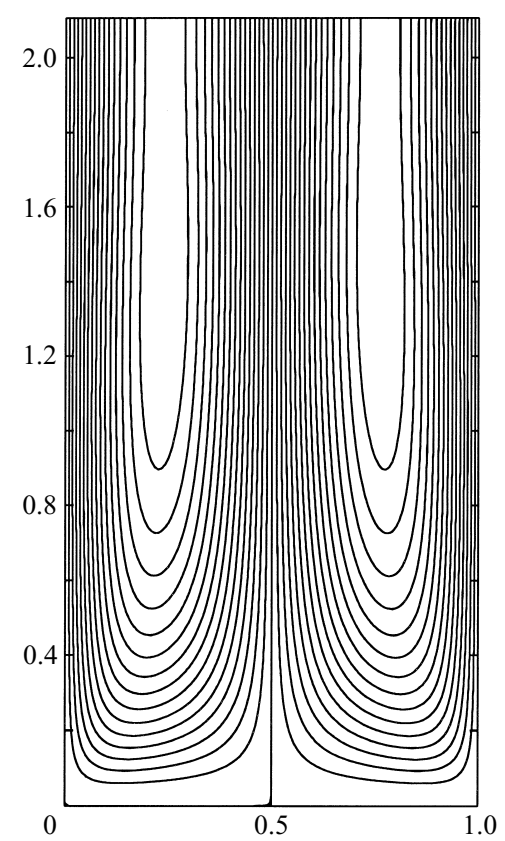

(b)

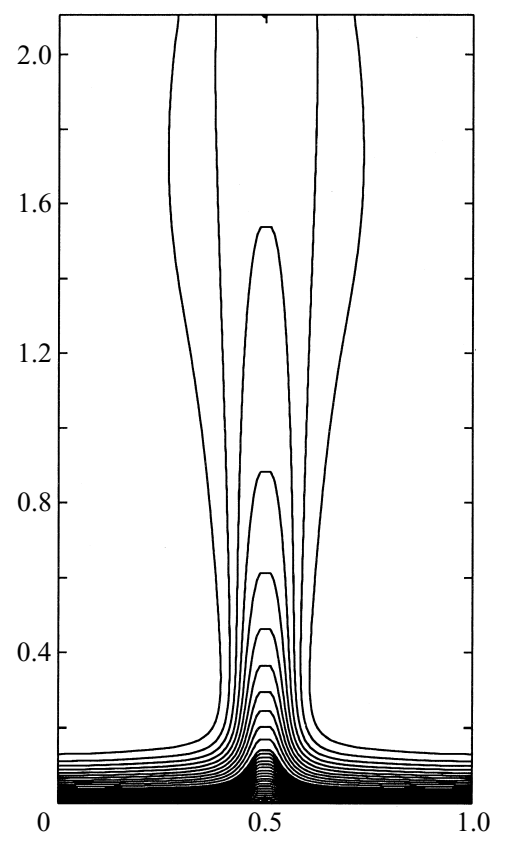

FIGURE 4. Streamlines $(a)$ and isotherms $(b)$ for the single plume obtained from the computation: $G r_{L}^{1 / 3}=29.24$ and $P r=6.0$.

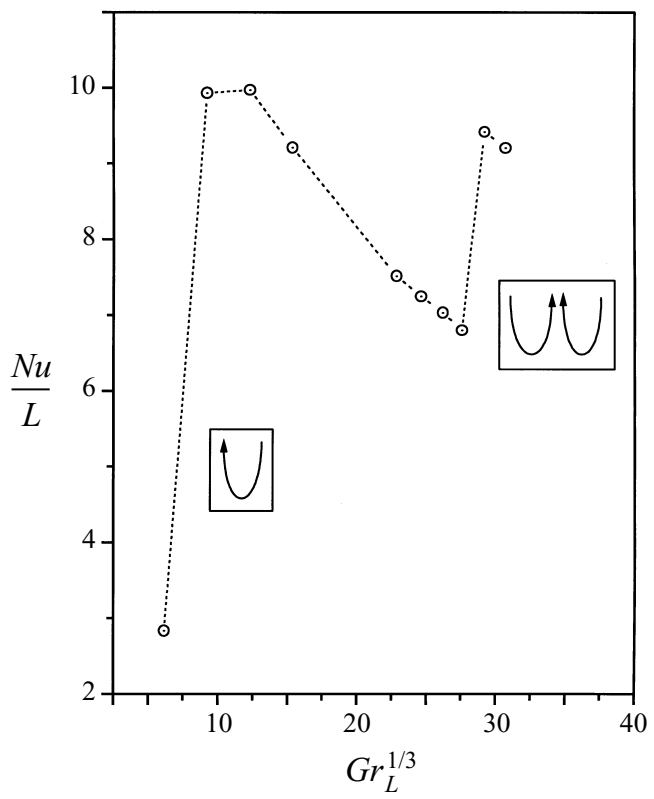

FIGURE 5. Variation of average heat flux, $N u / L$, with non-dimensional cavity length, $G r_{L}^{1 / 3}$, for $\operatorname{Pr}=6.0$. The jump in $N u / L$ at $G r_{L}^{1 / 3} \simeq 29$ corresponds to transition from half a plume to one plume in the computational domain. 
(a)

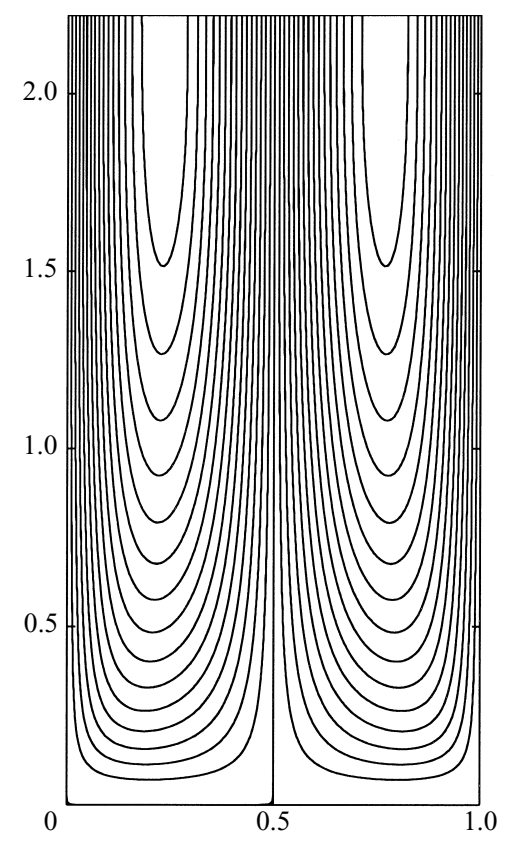

(b)

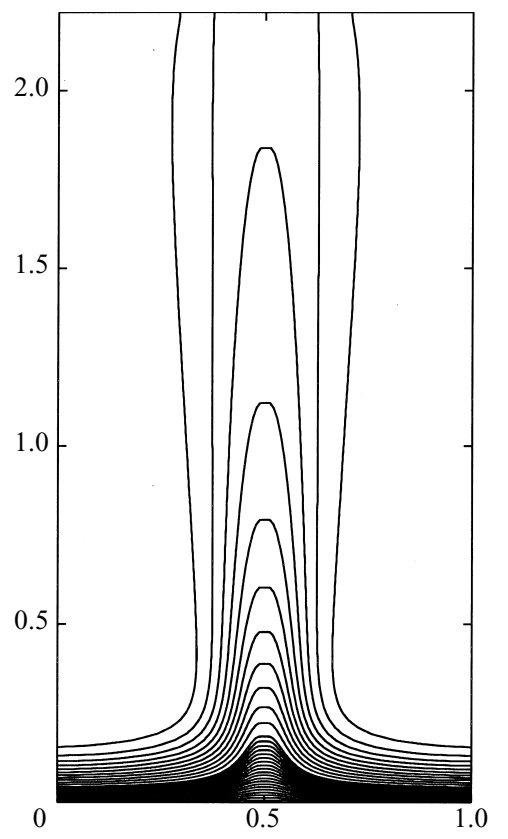

FIgURE 6. Same as figure 4 except $G r_{L}^{1 / 3}=54.74$ and $\operatorname{Pr}=0.7$.

at which the transition from a half-plume to one-plume takes place as the plume spacing; for $\operatorname{Pr}=6.0$, thus

and using equation (6) we get

$$
R a_{\lambda_{c}}^{1 / 3}=53
$$

$$
R a_{\Delta_{c}}=4050
$$

using $\eta_{\Delta}=3.0$. Compare these values to $R a_{\Delta_{c}}=2932$ and $R a_{\lambda_{c}}^{1 / 3}=51$ obtained in the previous section.

According to the numerical solution any plume spacing between 0 and $2 \lambda_{c}$ is possible. Our choice of spacing equal to $\lambda_{c}$ is exactly in between these two extremes. Experimentally a range of plume spacings is observed. In turbulent Rayleigh-Bénard convection experiments the presence of a larger clear region (no plume) usually results in the birth of a plume (Theerthan \& Arakeri 1994) consistent with the finding of maximum plume spacing in the present simulation. It may be noted that the variation in heat transfer rates for plume spacings between $\lambda_{c}$ and $2 \lambda_{c}$ is only about $25 \%$. As we shall see in the results below a change in plume spacing also does not significantly change the profiles of $\langle T\rangle,\left\langle T^{\prime 2}\right\rangle$ and the velocity fluctuations.

Similar observations are made for $\mathrm{Pr}=0.7$. Figure 6 shows the streamlines and isotherms for $\mathrm{Pr}=0.7$. Note that the thermal boundary layer thickness and plume width are larger compared to the $P r=6.0$ case.

Figure 7 shows the $N u / L$ curve for $\operatorname{Pr}=0.7$. For $P r=0.7$ we take

$$
R a_{\lambda_{c}}^{1 / 3}=48
$$

and using equation (6) we get

$$
R a_{\Delta_{c}}=5221
$$




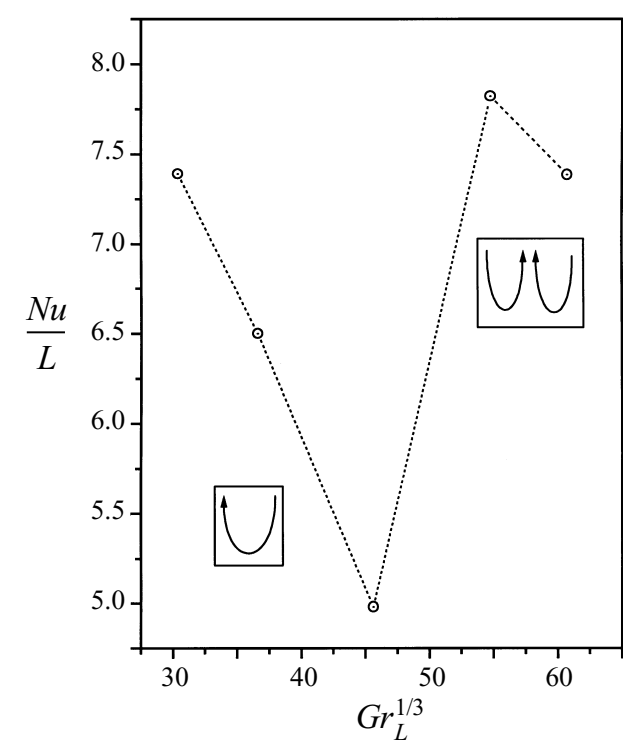

FIGURE 7. Same as figure 5 except $P r=0.7$. The jump in $N u / L$ occurs at $G r_{L}^{1 / 3} \simeq 54$.

using $\eta_{\Delta}=5.2$. These values compare well with $R a_{\Delta_{c}}=6634$ and $R a_{\lambda_{c}}^{1 / 3}=52$ obtained in the previous section. We use the values of $R a_{\lambda_{c}}$ given above in equations (35) and (37) for all subsequent calculations.

\section{Results}

\subsection{Nusselt number}

From equation (9) and using the values for $R a_{\lambda_{c}}$ in (35) and (37) we obtain the $N u-R a$ correlation for Rayleigh-Bénard convection:

$$
\begin{aligned}
N u & =\frac{\langle q\rangle D}{2 T_{w} k_{f}}=-\frac{5 \times 2^{1 / 15}}{6} H^{\prime}(0) R a_{\lambda_{c}}^{-2 / 15} \operatorname{Pr}^{-1 / 5} \operatorname{Ra}^{1 / 3} \\
& =0.073 R a^{1 / 3}(\operatorname{Pr}=6.0) \\
& =0.071 R a^{1 / 3}(\operatorname{Pr}=0.7) .
\end{aligned}
$$

The Nusselt number values from the numerical solution are slightly lower:

$$
\begin{aligned}
& N u=0.067 R a^{1 / 3}(\operatorname{Pr}=6.0), \\
& N u=0.0575 R a^{1 / 3}(\operatorname{Pr}=0.7) .
\end{aligned}
$$

The experimental correlation from Globe \& Dropkin (1959) gives

$$
\begin{aligned}
N u & =0.069 R a^{1 / 3} \operatorname{Pr}^{0.074} \\
& =0.0788 R a^{1 / 3}(\operatorname{Pr}=6.0) \\
& =0.0672 R a^{1 / 3}(\operatorname{Pr}=0.7) .
\end{aligned}
$$

It is not surprising that the three Nusselt numbers are not very different considering that the plume spacing calculated using the Globe \& Dropkin (1959) correlation is very similar to the plume spacing obtained from the numerical simulation. The more accurate $N u \sim R a^{2 / 7}$ correlations do not give significantly different $N u$ values in the Rayleigh number range of our interest. 
For convection over horizontal plates, $N u_{h p}$ is traditionally based on $T_{w}$. The numerical constants in (40) to (43) respectively become $0.184,0.179,0.169,0.145$. The experimental correlations (Fuji \& Imura 1972; Kitamura \& Kimura 1995) give about $25 \%$ lower value of $N u_{h p}$ compared to the model prediction.

\subsection{Plume spacing}

In this subsection we compare the experimental values of plume spacing reported in the literature with the plume spacing predicted by our model. We denote the plume spacing obtained from experiments by $\lambda_{p}$. Plume spacing taken from our numerical simulation results (35) and (37) and repeated below for the two $P r$ values are

$$
\begin{aligned}
\frac{\lambda_{c}}{Z_{w}} & =R a_{\lambda_{c}}^{1 / 3} \\
& =53(\operatorname{Pr}=6.0) \\
& =48(\operatorname{Pr}=0.7) .
\end{aligned}
$$

The plume spacing, $\lambda_{c}$, normalized by the characteristic length can be derived as

$$
\begin{aligned}
\frac{\lambda_{c}}{D} & =2^{1 / 3} R a_{\lambda_{c}}^{1 / 3}\left[g \beta\left(2 T_{w}\right) D^{3} / v \alpha\right]^{-1 / 3}=2^{1 / 3} R a_{\lambda_{c}}^{1 / 3} R a^{-1 / 3} \\
& =67 R a^{-1 / 3}(P r=6.0) \\
& =60 R a^{-1 / 3}(P r=0.7) .
\end{aligned}
$$

Note that $\lambda_{c}$ is independent of $D$ which is to be expected from such a model.

Husar \& Sparrow (1968) have visualized planforms of convection in water over different shaped horizontal heated plates. At the higher Rayleigh numbers the pictures near the centre of the plate show a cell-like structure. The cube root of Rayleigh number of the plume spacing, $R a_{\lambda_{p}}^{1 / 3}$ in this region (centre of the plate) is 70 (their figure $2 b$ ) and 45 (their figure $4 a$ ) for the two cases where a clear cell structure is visible (compare with the value of 53 predicted by the present model). In the other cases reported there is substantial edge effect and no comparison can be made. In our experiments (figure 1) $R a_{\lambda_{p}}^{1 / 3} \simeq 70$.

Tamai \& Asaeda (1984) in their experiments measured the average length of the bursting lines that formed a polygonal cell type structure. From this a representative horizontal scale for the bursting lines, $l$, was determined as

$$
\begin{array}{ll}
l / D=64.2 R a_{f}^{-1 / 4}, & 10^{6}<R a_{f}<10^{8}, \\
l / D=14.0 R a_{f}^{-1 / 6}, & 10^{8}<R a_{f}<10^{11} .
\end{array}
$$

$R a_{f}$ is the Rayleigh number based on the heat flux, instead of $\Delta T$. The two Rayleigh numbers are related through the expression $R a_{f}=N u R a$. Writing (53) in terms of $R a$, the $l / D \simeq 121 R a^{-1 / 3}$ compared to $67 R a^{-1 / 3}$ predicted by our model. Note that $l$ represents length of the plumes which is generally larger than plume spacing.

In the case of air $(P r=0.7)$, Deardorff \& Willis (1967) obtained the horizontal scale of motion by measuring the distance between the peaks of the correlation of temperature and the vertical velocity $\left(C_{w t}\right)$ at mid-height of the cell. They found that $L / D \approx 5.0$ for primary intensity maxima and $0.7<L / D<1.3$ for a secondary maxima, both at the mid-cell. At higher Rayleigh numbers there is a tendency for the primary maxima to shift towards larger wavelengths. We believe that primary maxima, with a higher intensity, are due to the large-scale flow and the secondary maxima, with a weaker intensity, are due to the wall plumes, i.e. $\lambda_{p} \sim$ spacing of the 
secondary maxima. For $\operatorname{Pr}=0.7$ we get from our analysis $\lambda_{c} / D \simeq 0.3$ to 1.31 for Rayleigh numbers in the range $\left(10^{7}>R a>10^{5}\right)$ which is close to the secondary maxima spacing observed by Deardorff \& Willis (1967). (In terms of non-dimensional spacing their value of $R a_{\lambda_{p}}^{1 / 3}$ is around 100 for the range of Rayleigh numbers $6.3 \times 10^{5}$ to $1.0 \times 10^{7}$ covered in their experiments.) It should be noted that the measurements of Deardorff \& Willis (1967) were at the mid-plane of the cell and not near the wall. The near-wall scales retain their identity at the midplane as evidenced by flow visualization pictures and the recent DNS by Kerr (1996) at these moderate $R a$.

At other Prandtl numbers, equation (23) predicts that the Rayleigh number based on plume spacing is essentially independent of $\mathrm{Pr}$. Measurements of plume spacing in experiments covering a wide range of Prandtl numbers are necessary to confirm this result.

\subsection{Mean and r.m.s. distributions}

The model can be used to obtain the distributions of mean temperature, r.m.s. of the temperature fluctuations and r.m.s. of the fluctuations of horizontal and vertical components of velocity. In this section we compare the distributions obtained from our model using the two methods of solution and the measured distributions reported in the literature. We look at two fluids, namely, water $(P r=6.0)$ and air $(P r=0.7)$, for which extensive data are available. All the quantities are non-dimensionalized by the wall variables $U_{w}, Z_{w}$ and $T_{w}$ (see (16) to (21)). For $P r=6.0$ the experimental data cover a range of $R a$ from $1.28 \times 10^{7}$ to $2.52 \times 10^{9}$, and for $P r=0.7$ the range is $2.5 \times 10^{6}$ to $1 \times 10^{8}$. The experimental data, except those of Adrian et al. (1986), Prasad \& Gonuguntla (1996) and Townsend (1959), are for Rayleigh-Bénard convection. In the experiments of Adrian and those of Prasad the top surface is insulating and in those of Townsend the top is open to the atmosphere. We have not come across any measurements of temperature or velocity distributions for convection over horizontal plates.

Figure 8 shows the comparison between experimental observation and the similarity calculations, for $P r=6.0$. The similarity solution has two contributions - one from the boundary layer and the other from the plume, labelled as B and P respectively in the figure. Figure 9 shows the corresponding plots for the numerical calculations.

The mean temperature distributions (figures $8 a$ and $9 a$ ) predicted by the model using the two methods agree well with the experimental data. The mean temperature asymptotes to a small non-zero value at large $Z / Z_{w}$ because of the contribution from the plume.

The r.m.s. of the temperature fluctuations calculated from the similarity solutions is shown in figure $8(b)$. The plume solution will be valid only beyond $Z / Z_{w} \simeq 15$. The experimental data show a maximum of about 0.15 in temperature fluctuations at $Z / Z_{w} \simeq 10$. Calculations from the model by both the methods agree well with the experimental data. Both the peak value and its position are well predicted. It is clear that the characteristic bulge in the r.m.s. temperature fluctuations is due to the boundary layer flow.

The nature of the r.m.s. horizontal velocity fluctuations shown in figures $8(c)$ and $9(c)$ predicted by the model is different from experimental observations. The predicted fluctuations go to zero at some distance from the wall, but in the experiments they go to some non-zero but nearly constant value. The horizontal velocity fluctuations away from the wall are caused by the large-scale motion which is not accounted for in the present model. However, these large-scale velocity fluctuations seem only to play a passive role as far as the wall layer is concerned, at least at moderate 

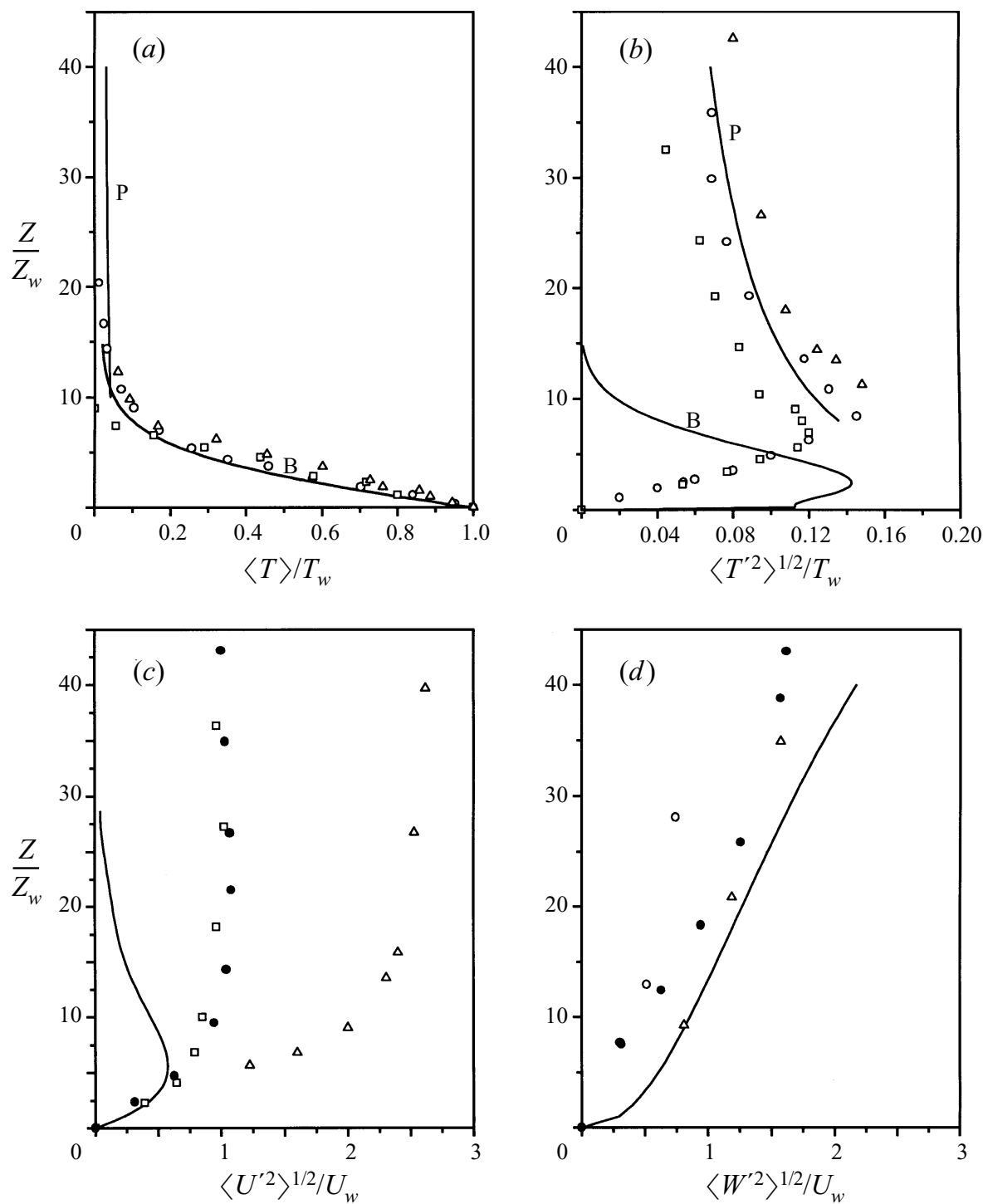

FiguRE 8. Results from similarity calculation (solid line) for $\mathrm{Pr}=6.0$. Symbols indicate data from experiments. (a) Average temperature: $\bigcirc, R a=1.28 \times 10^{7}$ (CG); $\square, 1.1 \times 10^{9}$ (TBL); $\triangle, 2.04 \times 10^{9}(\mathrm{TM})$; (b) r.m.s. temperature fluctuations: $\bigcirc, R a=3.21 \times 10^{8}(\mathrm{SG}) ; \triangle, 1.0 \times 10^{9}$ (AFB); $\square, 1.1 \times 10^{9}$ (TBL); (c) r.m.s. horizontal velocity fluctuations: $\triangle, R a=1.0 \times 10^{9}$ (AFB); $\square, 1.1 \times 10^{9}$ (TBL);,$R a=2 \times 10^{7}(\mathrm{PG}) ;(d)$ r.m.s. vertical velocity fluctuations: $\bigcirc, R a=2.52 \times 10^{9}$ (GG); $\triangle, 1.0 \times 10^{9}$ (AFB);,$R a=2 \times 10^{7}$ (PG). B refers to the boundary layer part ((16) to (18)) and P refers to the plume part ((19) to (21)). The experimental results are taken from the references: Chu \& Goldstein (1973) (CG), Somerscales \& Gazda (1969) (SG), Adrian et al. (1986) (AFB), Tilgner et al. (1993) (TBL), Garon \& Goldstein (1973) (GG), Tanaka \& Miyata (1980) (TM) and Prasad \& Gonuguntla (1996) (PG)

Rayleigh numbers. It seems the addition of an outer velocity and the calculated inner velocity profile will give the experimentally observed $\left\langle U^{\prime 2}\right\rangle^{1 / 2}$ distribution. As discussed in the introduction and below, at what Rayleigh number the outer flow starts affecting the wall region and the related issue of transition to hard turbulence are still open questions. However, it is probably important to note that Adrian et al.'s 

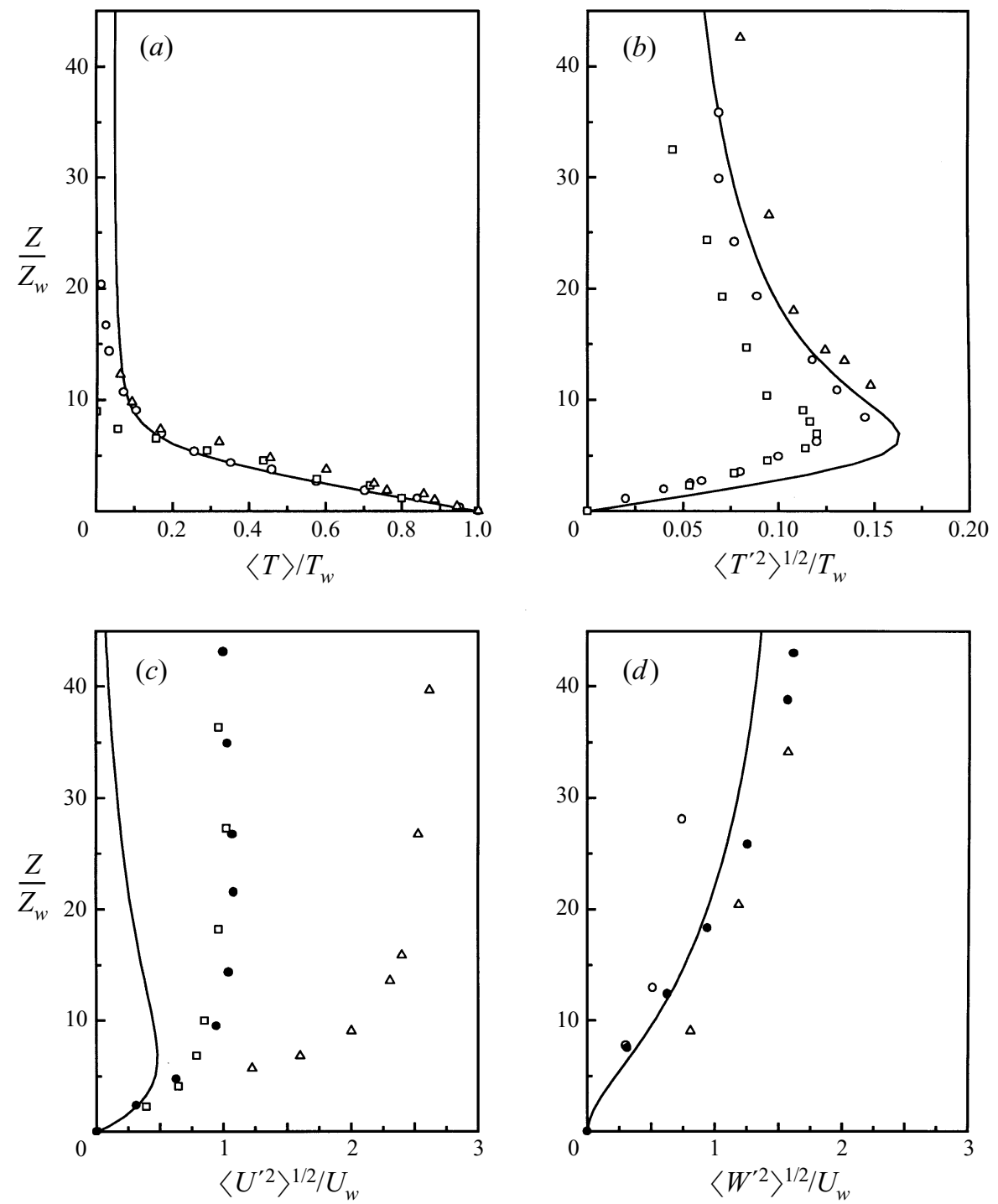

FIGURE 9. As figure 8 but showing results from the numerical calculation (solid line). For symbols see figure 8 .

(1986) experiments were unsteady, non-penetrative convection in wide layers where the large scale flow is likely to be different from that in Rayleigh-Bénard convection.

The predicted r.m.s. of vertical velocity fluctuations shown in figures $8(d)$ and $9(d)$ show distributions similar to that observed in the experiments. The smaller values obtained in the experiments of Garon \& Goldstein (1973) can be due to the lower aspect ratio $(\simeq 2.5)$. Clearly more experimental data are needed before any conclusion can be drawn.

Calculations for $P r=0.7$ (figures 10 and 11) show similar trends as for $P r=6$. The prediction of the r.m.s. temperature fluctuations distribution, surprisingly, almost coincides with the measurements of Townsend (1959). The vertical velocity fluctuations compare very well with the measurements of Deardorff \& Willis (1967a).

Figure 12 shows the proportions of conductive $\left(-k_{f} \partial T / \partial Z\right)$ and convective heat 

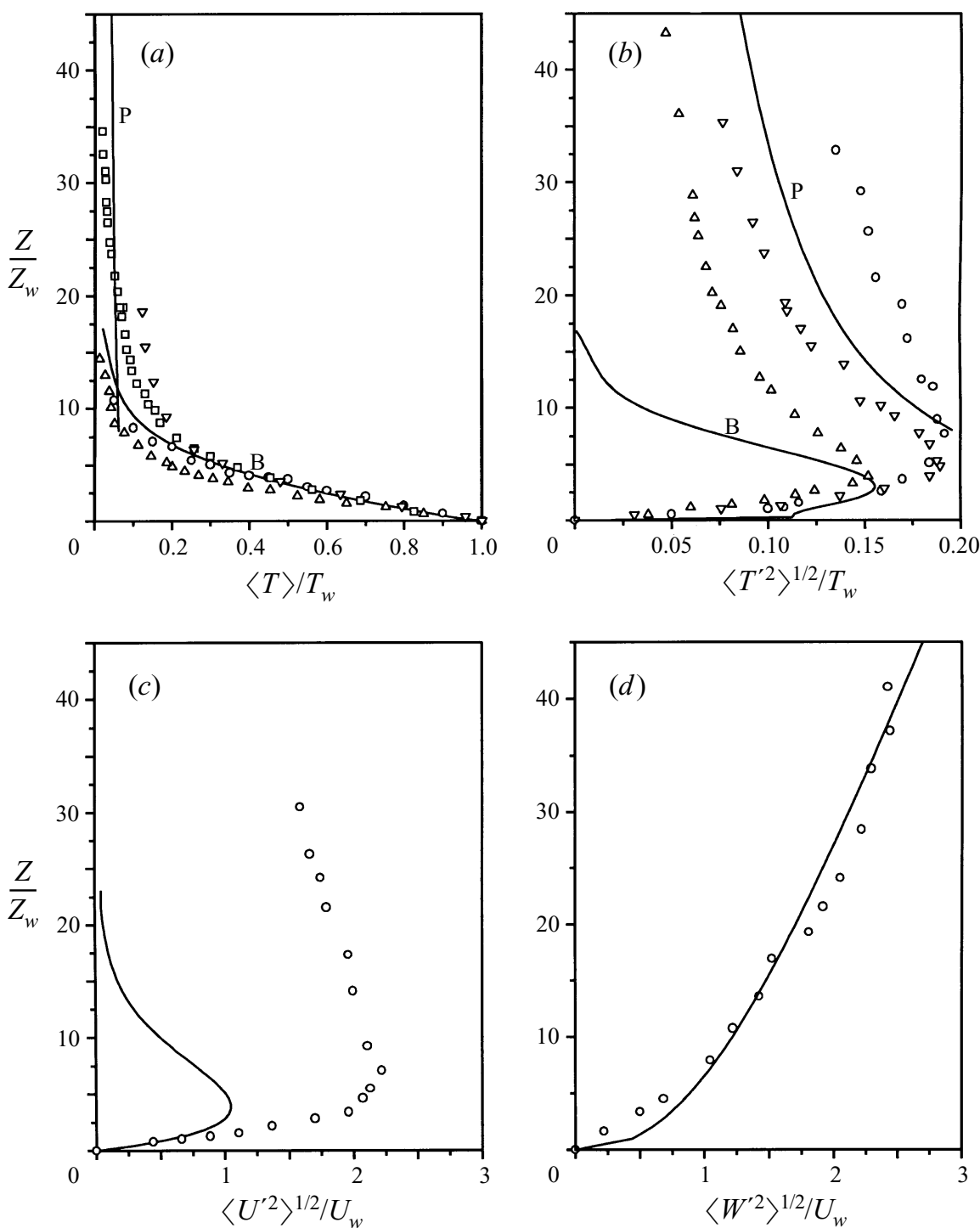

FiguRE 10. Results from similarity calculation (solid line) for $\mathrm{Pr}=0.7$. Symbols indicate data from experiments. (a) Average temperature: $\bigcirc, R a=2.5 \times 10^{6}(\mathrm{DWa}) ; \triangle, 4.8 \times 10^{7}(\mathrm{BTL}) ; \nabla, 1.0 \times 10^{8}$ (T); $\square, 7.8 \times 10^{7}(\mathrm{DWb})$; (b) r.m.s. temperature fluctuations: $\bigcirc, R a=2.5 \times 10^{6}(\mathrm{DWa}) ; \nabla, 1.0 \times 10^{8}$ (T); $\triangle, 4.8 \times 10^{7}$ (BTL); (c) r.m.s. horizontal velocity fluctuations: $\bigcirc, R a=2.5 \times 10^{6}$ (DWa); (d) r.m.s. vertical velocity fluctuations: $\bigcirc, R a=2.5 \times 10^{6}$ (DWa). B refers to boundary layer part ((16) to (18)) and P refers to the plume part ((19) to (21)). The experimental results for air are taken from Deardorff \& Wills (1967a) (DWa), Belmonte et al. (1993) (BTL), Deardorff \& Willis (1967b) $(\mathrm{DWb})$ and Townsend (1959) (T).

transfer for $\operatorname{Pr}=6.0$ from the numerical computation. It is clear, for $Z / Z_{w}>15$ almost all of the heat transfer is due to convection by the plumes. Further away from the wall at least part of the convection will be due to the outer-scale eddies. This picture is similar to wall-bounded turbulent flows where momentum transfer switches from the purely viscous at the wall to the 'large'-scale turbulence via the intermediate sublayer vortices. 

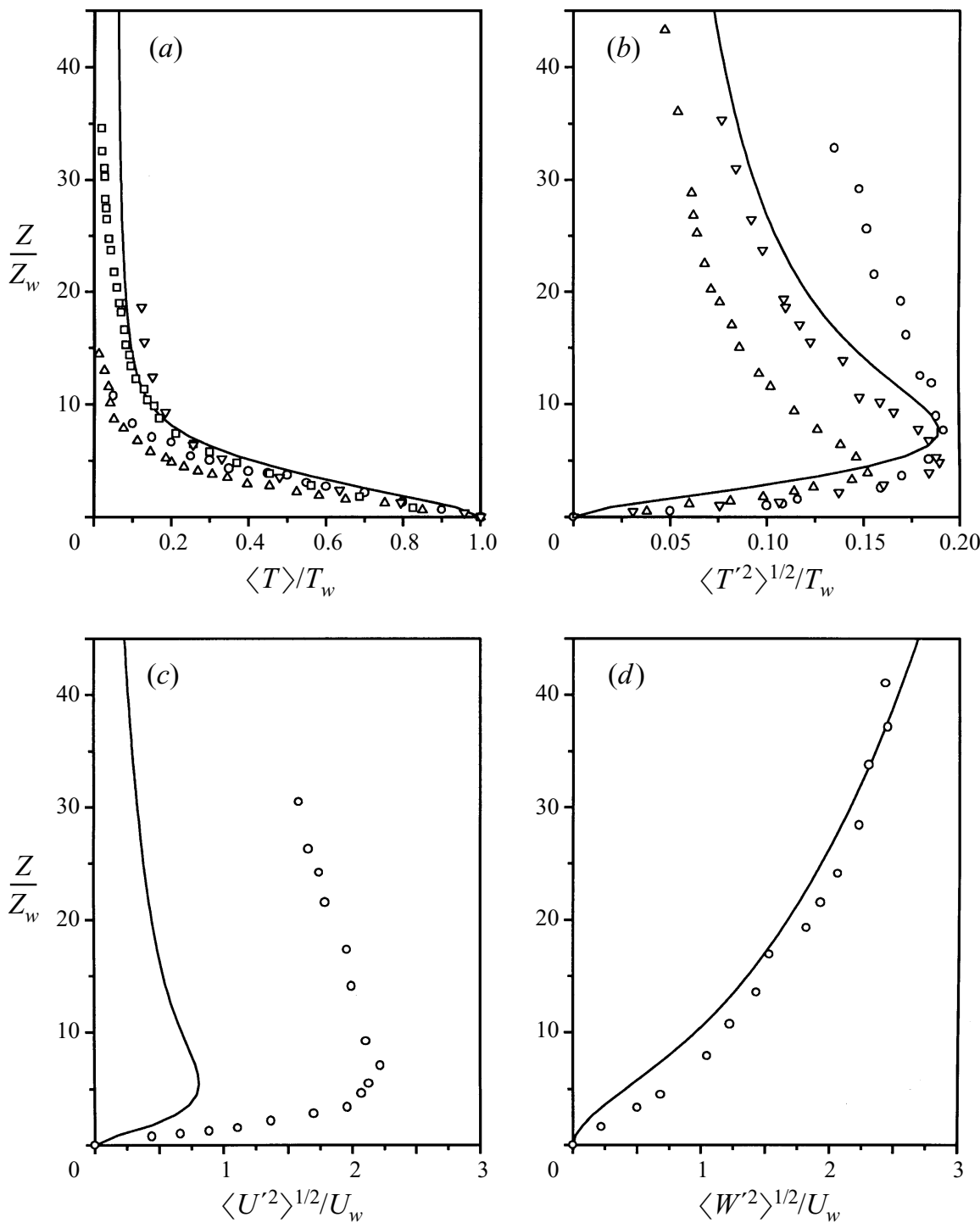

FIGURE 11. As figure 10 but showing results from the numerical calculation (solid line). For symbols see figure 10 .

A major drawback of the present model is that it fails to predict the $R a^{2 / 7}$ dependence of Nusselt number in Rayleigh-Bénard convection. Any improvement of the model will have to await a clearer understanding of the outer flow-inner flow coupling. One way to bypass the issue is to use heat flux as the independent variable instead of the temperature difference, that is to write expressions (16) to (21) in terms of Townsend's variables using (31) to (33). Figures 9 and 11 redrawn in terms of Townsend's variables are shown in figures 13 and 14. The near-wall distributions of the various quantities for a given $R a$ may then be obtained using the $N u \sim R a^{2 / 7}$ correlation for Rayleigh-Bénard convection and appropriate correlations for other configurations. Comparison of figures 13 and 14 shows the $\operatorname{Pr}$ dependence for the 


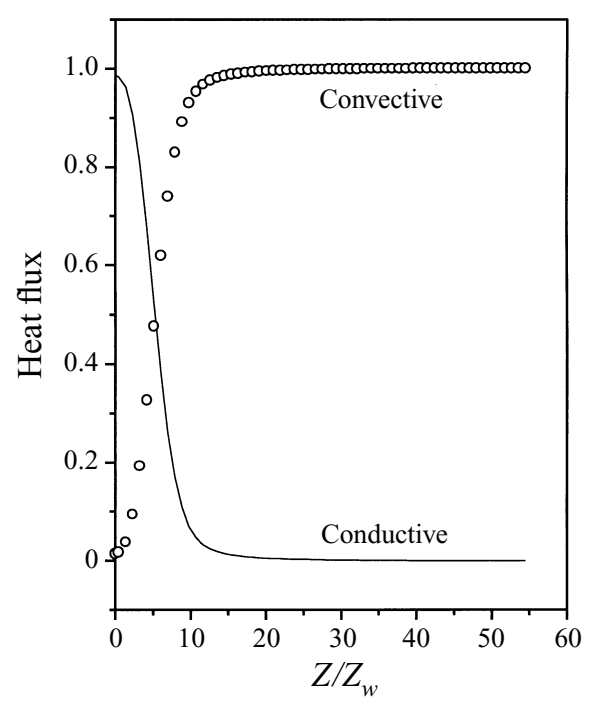

Figure 12. Proportions of conductive $\left(-k_{f} \partial T / \partial Z\right)$ and convective heat transfer obtained from numerical simulation for $\mathrm{Pr}=6.0$.

distributions of the various quantities. The plume spacing in terms of heat flux is given by (26).

In all the above calculations we have assumed the plume spacing to be constant $=$ $\lambda_{c}$ given by the relations (35) and (37). In experiments a range of plume spacings are observed. To get an idea of the effect of plume spacing we plot distributions of the various quantities for a range of plume spacings between $\lambda_{c}$ and $2 \lambda_{c}$ for $\operatorname{Pr}=0.7$ (figure 15). As noted in $\S 3$ the variation in heat flux is about $25 \%$. The effect is largest for the r.m.s. of vertical velocity where the variation is about $50 \%$. However, the variations in the various quantities due to plume spacing change is about the same as the experimental scatter.

\section{Discussion}

In spite of the real flow field being unsteady and three-dimensional the simple twodimensional model appears to capture most of the near-wall dynamics of turbulent natural convection over a heated horizontal surface. In particular, the plume spacing seems to be determined by a criticality condition, and both the boundary layer and plume regions need to be considered to describe the dynamics. The model may be considered to be a more accurate method of calculation than the more simple minded approaches of, say, equating the buoyancy and viscous forces as was done in the past. The model may be extended by considering the flow to be an ensemble of randomly oriented line plumes (akin to the ensemble of randomly oriented vortices for models of isotropic turbulence) instead of the parallel array of plumes we have considered. With such a model higher-order statistics and two-point correlations can be calculated.

It is useful to estimate the range of Rayleigh numbers under which our model is likely to be valid in Rayleigh-Bénard convection. The wall layer dynamics seem to extend to a non-dimensional height $Z / Z_{w} \simeq 50$ or equivalently a Rayleigh number based on height to about $1.25 \times 10^{5}$. Thus our model, which assumes plumes from the opposite wall have no role to play, may be expected to be valid when the non- 

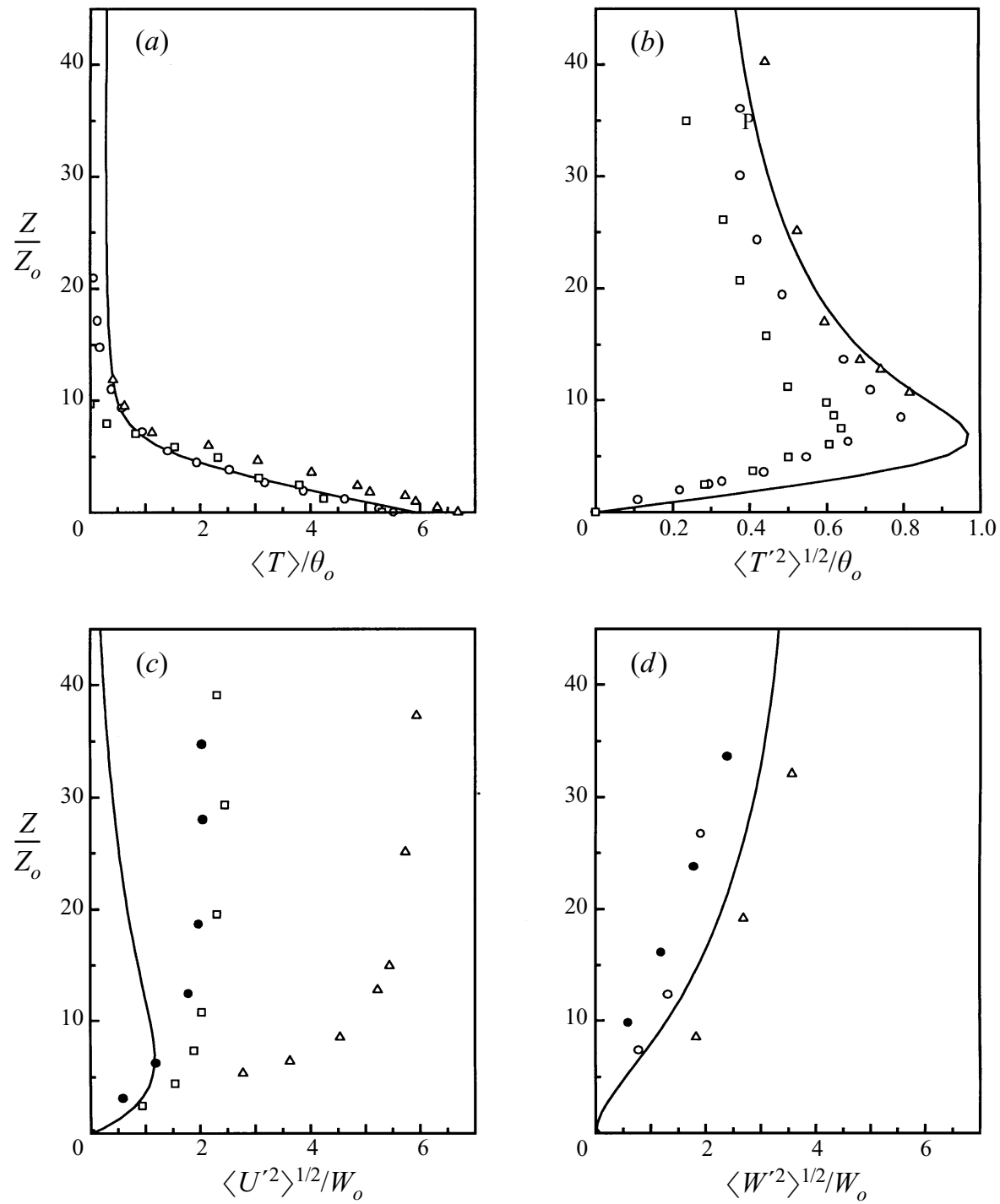

FIGURE 13. Figure 9 redrawn in terms of Townsend's variables.

dimensional distance between the plates $D / Z_{w}>100 . D / Z_{w}=100$ corresponds to $R a=2 \times 10^{6}$. Thus it is also clear that effects of viscosity are felt in the centre of the cell even at $R a$ as high as $10^{6}$. Horizontal velocity fluctuations exist across the height of the convection cell. These fluctuations in the core region away from the plate are presumably a result of interaction of plumes from the opposite walls and from general turbulent mixing. These fluctuations are felt right up to the wall but as noted earlier seem to play a passive role in the near-wall dynamics.

As the Rayleigh number increases the outer velocity scale $W_{*}$ increases with respect to the inner velocity scale $U_{w}$. Thus at very large Rayleigh numbers the outer flow should start influencing the near-wall dynamics. When this will happen is still unresolved. As mentioned earlier, most of the experiments at high $\operatorname{Ra}\left(\geqslant 10^{9}\right)$ have been in cells with aspect ratios of order one. The geometry then imposes a single 

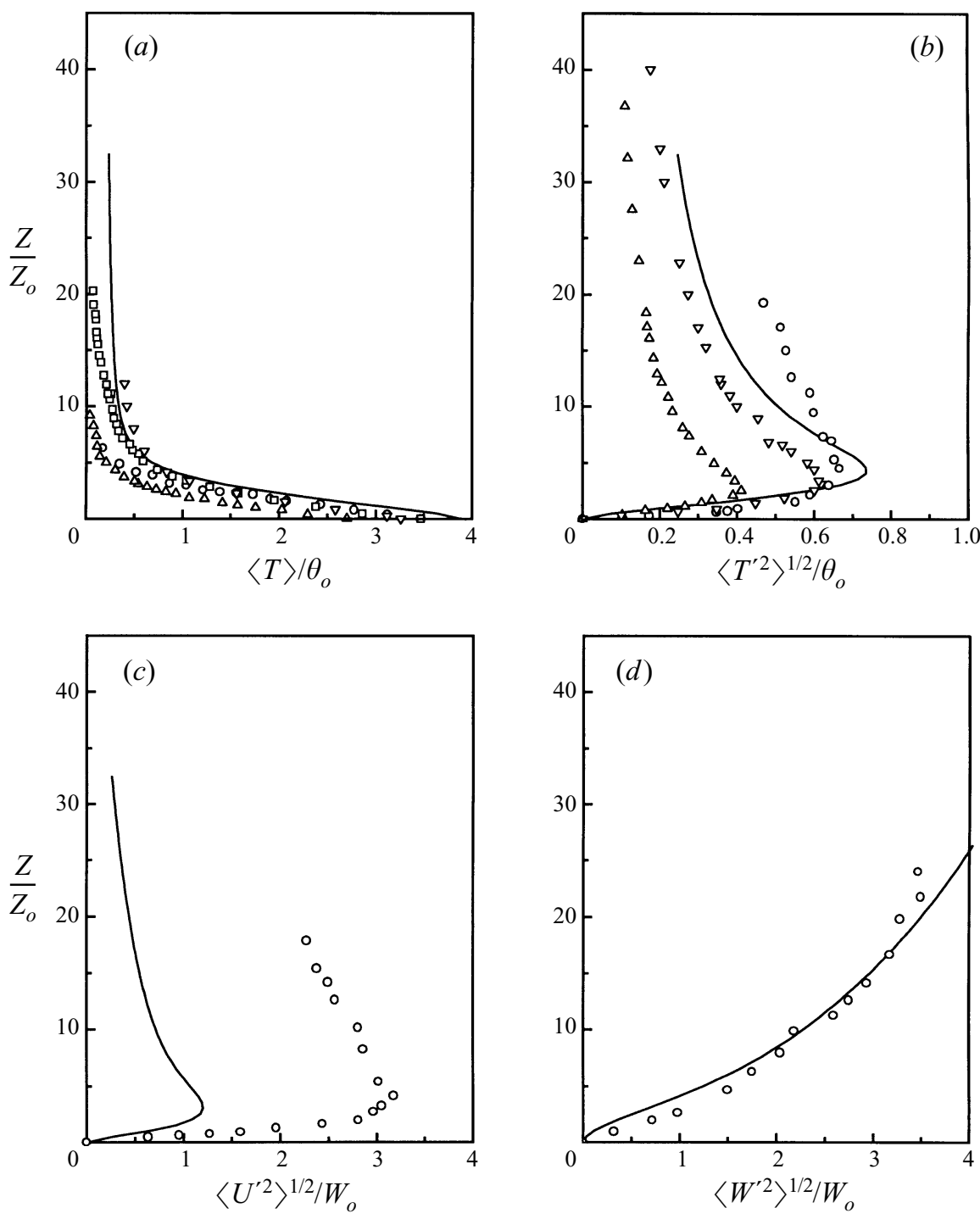

FIGURE 14. Figure 11 redrawn in terms of Townsend's variables.

large-scale roll which sweeps the near-wall plumes. Experiments at high Rayleigh numbers are needed to tell us whether energetic coherent rolls scaling with the height of the cell will persist even at high aspect ratios. However, we expect that our model will be valid at least for Rayleigh numbers in the range of $10^{7}$ to $10^{9}$.

Flow structures away from the wall layer in convection over a single horizontal surface and in Rayleigh-Bénard convection will be different as shown in figure 2. In the former there will be no impinging plumes from the opposite wall and large-scale flow will be that due to the turbulent boundary layer and the turbulent plume(s). On finite plates there is a distinct edge effect with longitudinal rolls extending some distance normal to the edge (Husar \& Sparrow 1968; Kallol Bera 1993). The flow away from the edges and near the surface, however, consists of line plumes and our model should be valid there. 

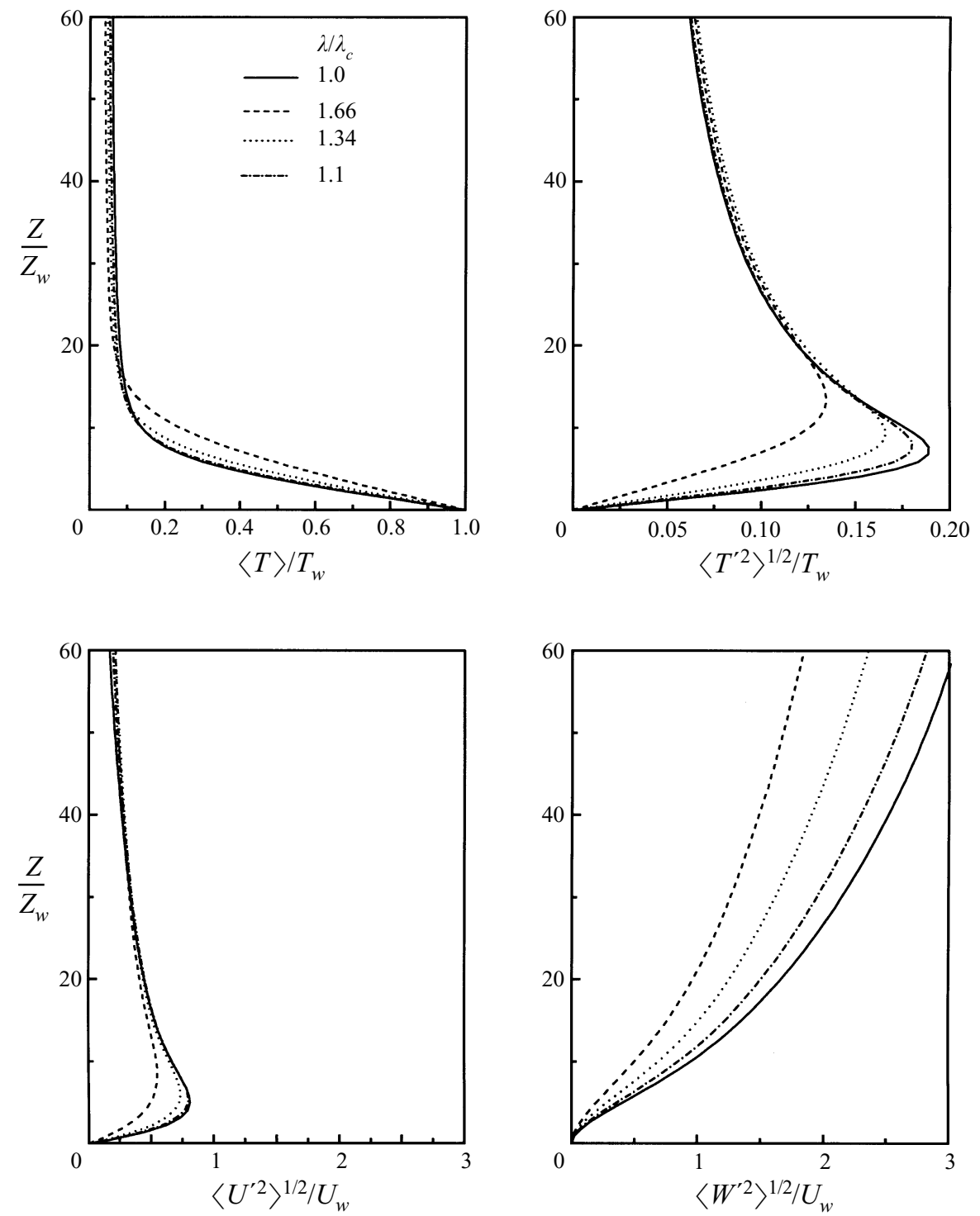

FIGURE 15. The various distributions plotted for a range of plume spacings between $\lambda_{c}$ and $2 \lambda_{c}$ for $\operatorname{Pr}=0.7$.

Another unresolved issue, briefly brought out in $\S 4.2$, is the appropriate Prandtl number dependence of the various scales. It would be desirable to obtain Prandtlnumber-independent distributions of average temperature, temperature fluctuations and the velocity fluctuations. We do not know whether this is indeed possible. For example expressions (16) to (21) for distributions of the various quantities in the boundary layer region and in the plume region show different and complicated Prandtl number dependences. Experiments with a large range of Prandtl numbers are needed to resolve some of these issues and also to ascertain whether $R a_{\lambda_{c}}$ is indeed nearly independent of $\mathrm{Pr}$ as predicted by the model.

The model and the analytical expressions provide a natural basis to obtain 'wall functions' to use in turbulence models to calculate natural convection flows over 
horizontal surfaces. It will also be useful for subgrid modelling near the wall in large-eddy-simulation (LES) calculations. Near-wall distributions of kinetic energy $(k)$, production of $k$ (which will be due to buoyancy), dissipation of $k$ and dissipation of temperature fluctuations may be calculated using the model.

It is of interest to point out here that the line-plumes structure is also observed in convection over the Earth's surface under 'still' (no wind) conditions. Of course, in this case the flow in the boundary layers will be turbulent. Extending the present model with the molecular diffusivities replaced by the turbulent (eddy) diffusivities is, an attractive, but perhaps far fetched, proposition. Qualitatively, however, the flow appears to be similar to the model we have proposed. It would be interesting to check whether in the atmospheric convection also the plume spacing is about five times the boundary layer thickness.

\section{Conclusions}

We have proposed a model which describes the near-wall dynamics of turbulent natural convection over a horizontal surface at moderate Rayleigh numbers. The model consisting of laminar boundary layer and an array of steady line plumes reproduces the distributions of mean temperature, r.m.s. temperature fluctuations and r.m.s. vertical velocity fluctuations close to the wall, reasonably well. The deviations observed in the case of r.m.s. horizontal velocity fluctuations is due to the largescale flow present in the experiments and which is not accounted for in the present model. However, as indicated earlier, these large-scale flows play only a passive role as far as the wall layer events are concerned, at least at these moderate $R a$. The average plume spacing predicted by the model is in reasonable agreement with the limited experimental data. More experiments are required to check the prediction that Rayleigh number based on plume spacing is around $1.4 \times 10^{5}$, nearly independent of Prandtl number.

\section{Appendix A. The boundary layer}

Rotem \& Classen (1969) give the distributions of horizontal velocity $F^{\prime}(\eta)$ and temperature $H(\eta)$ where $\eta$, the similarity variable, $=\left(Z / \frac{1}{2} \lambda_{c}\right) G r_{c}^{1 / 5}\left(X / \frac{1}{2} \lambda_{c}\right)^{-2 / 5}, X$ is horizontal distance from the leading edge and $Z$ is vertical distance.

The horizontal component of velocity is given by

$$
U=\left(\frac{v}{\lambda_{c}}\right) \operatorname{Ra}_{\lambda_{c}}^{2 / 5} \operatorname{Pr}^{-2 / 5}\left(\frac{X}{\lambda_{c}}\right)^{1 / 5} F^{\prime}(\eta)
$$

$F(\eta)$ is the stream function.

The r.m.s. of the horizontal velocity fluctuations using (2) is

$$
\left\langle U^{\prime 2}\right\rangle_{b}^{1 / 2}=\sqrt{5}\left(g \beta T_{w} v\right)^{1 / 3} R a_{\lambda_{c}}^{5 / 12} \operatorname{Pr}^{-5 / 12}\left(\frac{Z}{\lambda_{c}}\right)^{7 / 4}\left(\int_{\eta_{c}}^{\infty} \eta^{-9 / 2} F^{\prime 2}(\eta) \mathrm{d} \eta\right)^{1 / 2} .
$$

In the above equations $\eta_{c}$ is a function of $Z$ and $P r$ and the rest of the quantities are either constants or functions of $P r$. The subscript $b$ is used to indicate the contribution from the boundary layer. Note that the average horizontal velocity is zero with contributions from the two boundary layers cancelling each other exactly. 
The mean temperature profile in the boundary layer is obtained as

$$
\frac{\langle T\rangle_{b}}{T_{w}}=5\left(\frac{Z}{\lambda_{c}}\right)^{5 / 2} \operatorname{Ra}_{\lambda_{c}}^{1 / 2} \operatorname{Pr}^{-1 / 2} \int_{\eta_{c}}^{\infty} H(\eta) \eta^{-7 / 2} \mathrm{~d} \eta
$$

and the r.m.s. of the temperature fluctuations is obtained from

$$
\begin{aligned}
\left\langle T^{\prime 2}\right\rangle_{b}=T_{w}^{2}\left(5\left(\frac{Z}{\lambda_{c}}\right)^{5 / 2}\right. & R a_{\lambda_{c}}^{5 / 2} \operatorname{Pr}^{-5 / 2}\left(\int_{\eta_{c}}^{\infty} H^{2}(\eta) \eta^{-7 / 2} \mathrm{~d} \eta\right)^{1 / 2} \\
& \left.-25\left(\frac{Z}{\lambda_{c}}\right)^{5} R a_{\lambda_{c}} \operatorname{Pr}^{-1}\left[\int_{\eta_{c}}^{\infty} H(\eta) \eta^{-7 / 2} \mathrm{~d} \eta\right]^{2}\right) .
\end{aligned}
$$

Here, $H(\eta)=\theta=T / T_{w}$ is the non-dimensional temperature. The first part on the right-hand side is the square of the r.m.s. of the total temperature and the second part is the square of $\langle T\rangle$.

The integrals for $\left\langle U^{\prime 2}\right\rangle^{1 / 2},\langle T\rangle_{b}$ and $\left\langle T^{\prime 2}\right\rangle^{1 / 2}$ are evaluated numerically after finding a fit for the similarity functions, $H(\eta), F(\eta), \ldots$ etc. that appear in the integrals. The limit $\infty$ is replaced by $\eta_{\Delta}$, the edge of the boundary layer. This replacement is equivalent to the integral with respect to $X$ not starting at 0 but at the edge of the boundary layer for any $Z$.

\section{Appendix B. Plume}

The vertical velocity in a laminar buoyant two-dimensional plume is given by (Fuji 1963)

$$
W(\xi)=f^{\prime}(\xi) Z^{1 / 5}\left(g \beta \theta_{l}\right)^{2 / 5} v^{1 / 5},
$$

where $f^{\prime}(\xi)$ is the similarity function for the plume region and

$$
\xi=G r_{z}^{1 / 5}\left(\frac{X}{Z}\right) ; \quad G r_{z}=\left(\frac{g \beta}{v^{2}}\right) \theta_{l} Z^{3} .
$$

Now we obtain the relation between $\mathrm{d} \xi$ and $\mathrm{d} X$ as,

$$
\mathrm{d} \xi=\left(\frac{g \beta \theta_{l}}{v^{2}}\right)^{1 / 5} Z^{-2 / 5} \mathrm{~d} X .
$$

In all the above expressions, $Z$ is measured from the virtual origin of the plume. Calculation of the virtual origin of the plume is done by equating the mass flow rate in the plume to the combined mass flow rate in the two boundary layers at the points where the boundary layers turn to form the plume (see figure $3 b$ ). A brief calculation is presented below.

In the calculation of the plume characteristics we require a quantity (see equation (B 1))

$$
\theta_{l}=\frac{Q_{l}}{\rho C_{p} v} .
$$

Here $Q_{l}$ is the heat flux per unit plume length. In our case $Q_{l}=\langle q\rangle \lambda_{c}$, i.e. the heat flux from the two boundary layers on either side of the plume.

Substituting $\langle q\rangle$ (equation (8)) and $\lambda_{c}$ (equation (6)) in the expression for $Q_{l}$ and then in $\theta_{l}$ (equation (B 4)) we obtain

$$
\begin{aligned}
\theta_{l} & =\left[-\frac{10}{3} H^{\prime}(0) R a_{\Delta_{c}}^{1 / 2} \operatorname{Pr}^{-3 / 2} \eta_{\Delta}^{-3 / 2}\right] T_{w}, \\
& =\mathscr{C}(\operatorname{Pr}) T_{w},
\end{aligned}
$$


$\mathscr{C}(\mathrm{Pr})$ is the quantity in the bracket in equation (B 5) and is a function of $\mathrm{Pr}$. Substituting in equation (B 1) and taking the average with respect to $X$,

$$
\langle W\rangle=Z^{1 / 5}\left(g \beta T_{w}^{2 / 5}\right)^{2 / 5} \mathscr{C}^{2 / 5} v^{1 / 5} \frac{1}{\lambda_{c} / 2} \int_{0}^{\lambda_{c} / 2} f^{\prime}(\xi) \mathrm{d} X,
$$

where

$$
\xi_{c}=G r_{z}^{1 / 5}\left(\frac{\delta_{p} / 2}{Z}\right)
$$

$\delta_{p}$ is the plume width at any $Z$. Expanding $\mathscr{C}$ (equation (B 5)) and using equation (6) we get

$$
\langle W\rangle=\left(\frac{Z}{\lambda_{c} / 2}\right)^{3 / 5}\left(g \beta T_{w} v\right)^{1 / 3}\left[-\frac{10}{3} H^{\prime}(0)\right]^{1 / 5}\left[\left(R a_{\lambda_{c}} / 8\right)^{-7 / 75} \operatorname{Pr}^{-8 / 75}\right] \int_{0}^{\xi_{c}} f^{\prime}(\xi) \mathrm{d} \xi .
$$

The r.m.s. velocity is obtained in a similar way. Squaring (B 1) and taking the average, we obtain

$$
\left\langle W^{2}\right\rangle=Z^{2 / 5}\left(g \beta T_{w}\right)^{4 / 5} \mathscr{C}^{4 / 5} v^{2 / 5} \frac{1}{\lambda_{c} / 2} \int_{0}^{\lambda_{c} / 2} f^{\prime 2}(\xi) \mathrm{d} X .
$$

Substituting for $\mathrm{d} X$ in terms of $\mathrm{d} \xi$, and for $\mathscr{C}$ we get

$$
\left\langle W^{2}\right\rangle=\left[-\frac{10}{3} H^{\prime}(0)\right]^{3 / 5}\left(g \beta T_{w} v\right)^{2 / 3}\left(\frac{Z}{\lambda_{c} / 2}\right)^{4 / 5}\left[\left(R a_{\lambda_{c}} / 8\right)^{2 / 75} \operatorname{Pr}^{-49 / 150}\right]^{2} \int_{0}^{\xi_{c}} f^{\prime 2}(\xi) \mathrm{d} \xi .
$$

Now we calculate the virtual origin of the plume. The mass flow rate in the boundary layer is given by

$$
m_{b}=\rho \int_{0}^{\infty} U \mathrm{~d} Z
$$

Using (A 1) and (3),

$$
m_{b}=\rho v G r_{c}^{1 / 5} \int_{0}^{\eta_{v}} F^{\prime}(\eta) \mathrm{d} \eta
$$

where $\eta_{v}$ defines the edge of the velocity boundary layer. It is equal to 6 for $\operatorname{Pr}=6.0$ and 7 for $\operatorname{Pr}=0.7$ (Rotem \& Classen 1969).

The mass flow rate in the plume at a vertical location $Z$ from the virtual origin is given by

$$
m_{p}=\rho \int_{0}^{\lambda_{c} / 2} W \mathrm{~d} X
$$

Using (B 1) and (B 3), (B 14) becomes

$$
m_{p}=2 \rho\left(\frac{Z}{\lambda_{c} / 2}\right)^{3 / 5} G r_{c}^{1 / 5} v\left[-\frac{10}{3} H^{\prime}(0)\right]^{1 / 5}\left[\frac{R a_{\Delta_{c}}}{\eta_{\Delta}^{3}}\right]^{1 / 10} \operatorname{Pr}^{-3 / 10} \int_{0}^{\xi_{c}} f^{\prime}(\xi) \mathrm{d} \xi
$$

The virtual origin is obtained by equating $2 m_{b}=m_{p}$. If $Z / \frac{1}{2} \lambda_{c}$ obtained is greater than $\Delta_{c} / \frac{1}{2} \lambda_{c}\left(=\left[G r_{\Delta_{c}} / \eta_{\Delta}^{3}\right]^{-1 / 2}\right)$, then the virtual origin is below the horizontal surface. Calculation shows that the virtual origin lies about one-fifth of a boundary layer thickness, $\Delta_{c} / \frac{1}{2} \lambda_{c}$ above the surface. Therefore, we assume the virtual origin to lie on the surface. 
The temperature distribution (Fuji 1963) in the plume is

$$
T=\left(\frac{g \beta}{v^{2}}\right)^{-1 / 5} \theta_{l}^{4 / 5} Z^{-3 / 5} h(\xi),
$$

where $h(\xi)$ is the similarity function for temperature.

Substituting $\theta_{l}=\mathscr{C} T_{w}$ (equation (B 5)) we obtain the average temperature

$$
\langle T\rangle=\left(\frac{g \beta}{v^{2}}\right)^{-1 / 5} \mathscr{C} T_{w}^{4 / 5} Z^{-3 / 5} \lambda_{c} / 2^{-1} \int_{0}^{\lambda_{c} / 2} h(\xi) \mathrm{d} X .
$$

Using (B 3) we obtain

$$
\langle T\rangle=T_{w} G r_{c}^{-2 / 5} \mathscr{C}^{3 / 5}\left(\frac{Z}{\lambda_{c} / 2}\right)^{-1 / 5} \int_{0}^{\xi_{c}} h(\xi) \mathrm{d} \xi .
$$

Substituting for $G r_{c}$ (equation (6)) and $\mathscr{C}$ (equation (B 5)),

$$
\langle T\rangle=T_{w}\left[\left(R a_{\lambda_{c}} / 8\right)^{-7 / 25} \operatorname{Pr}^{-8 / 25}\right]\left[-\frac{10}{3} H^{\prime}(0)\right]^{3 / 5}\left(\frac{Z}{\lambda_{c} / 2}\right)^{-1 / 5} \int_{0}^{\xi_{c}} h(\xi) \mathrm{d} \xi .
$$

The r.m.s. temperature is obtained in a similar fashion by squaring the temperature distribution (equation (B 16)), averaging and taking the square root:

$$
\left\langle T^{2}\right\rangle^{1 / 2}=T_{w}\left[\left(R a_{\lambda_{c}} / 8\right)^{-4 / 25} \operatorname{Pr}^{-27 / 50}\right]\left[-\frac{10}{3} H^{\prime}(0)\right]^{7 / 10}\left(\frac{Z}{\lambda_{c} / 2}\right)^{-2 / 5}\left(\int_{0}^{\xi_{c}} h^{2}(\xi) \mathrm{d} \xi\right)^{1 / 2} .
$$

Then the r.m.s. of the temperature fluctuations is obtained using the relation

$$
\left\langle T^{\prime 2}\right\rangle_{p}=\left\langle T^{2}\right\rangle-\langle T\rangle^{2},
$$

the subscript $p$ denoting the plume contribution.

\section{REFERENCES}

Adrian, R. J., Ferreira, R. T. D. S. \& Boberg, T. 1986 Turbulent thermal convection in wide horizontal fluid layers. Exps. Fluids 4, 121-141.

AsaedA, T. \& WatANABE, K. 1989 The mechanism of heat transport in thermal convection at high Rayleigh numbers. Phys. Fluids A 1, 861-867.

Aubry, N., Holmes, P., Lumley, J. L. \& Stoen, E. 1988 The dynamics of coherent structures in the wall region of a turbulent boundary layer. J. Fluid Mech. 192, 115-173.

Belmonte, A., Tilgner, A. \& Libchaber, A. 1993 Boundary layer length scales in thermal turbulence. Phys. Rev. Lett. 70, 4067-4070.

Bernard, P. S., Thomas, J. M. \& Handler, R. A. 1993 Vortex dynamics and the production of Reynolds stress. J. Fluid Mech. 253, 385-419.

Brooke, J. W. \& Hanratty, T. J. 1993 Origin of turbulence-producing eddies in a channel flow. Phys. Fluids A 5, 1011-1022.

Castaing, B., Gunaratne, G., Heslot, F., Kadanoff, L., Libchaber, A., Thomae, S., Wu, X.-Z., ZaLeSKi, S. \& ZANETTI, S. 1989 Scaling of hard turbulence in Rayleigh-Bénard convection. J. Fluid Mech. 204, 1-30.

ChU, T. Y. \& Goldstein, R. J. 1973 Turbulent convection in a horizontal layer of water. J. Fluid Mech. 60, 141-159.

COLES, D. 1978 Coherent structures of Turbulent boundary layers. In AFOSR/Lehigh University Workshop (ed. C. R. Smith \& Ch. D. E. Abbott), p. 462.

DeArdorfF, J. W. 1970 Convective velocity and temperature scales for the unstable planetary boundary layer and Rayleigh convection. J. Atmos. Sci. 27, 1211-1213. 
DeardorfF, J. W. \& Willis, G. E. 1967a Investigation of turbulent thermal convection between horizontal plates. J. Fluid Mech. 28, 675-704.

Deardorff, J. W. \& Willis, G. E. $1967 b$ The free convection temperature profile. Q. J. R. Met. Soc. 93, 166-175.

De Vahl Davies, G. \& Jones, I. P. 1983 Natural convection in a square cavity, a comparison exercise. Intl J. Numer. Meth. Fluids 3, 227-248.

FuJI, T. 1963 Theory of the steady laminar natural convection above a horizontal line source and a point heat source. Intl J. Heat Mass Transfer 6, 597-606.

Fuj, T. \& ImURA, H. 1972 Natural convection heat transfer from a plate with arbitrary inclination. Intl J. Heat Mass Transfer 15, 755-767.

Garon, A. M. \& Goldstein, R. J. 1973 Velocity and heat transfer measurements in thermal convection. Phys. Fluids 16, 1818-1825.

Globe, S. \& Dropkin, D. 1959 Natural convection heat transfer in liquids confined by two horizontal plates and heated from below. Trans. ASME J. Heat Transfer 81, 24-28.

Gluckman, B. J., Willaime, H. \& Gollub, J. P. 1993 Geometry of Isothermal and Isoconcentration surfaces in thermal turbulence. Phys. Fluids A 5, 647-661.

Goldstein, R. J. \& Volino, R. J. 1995 Onset and development of natural convection above a suddenly heated horizontal surface. Trans ASME J. Heat Transfer 117, 808-821.

Howard, L. N. 1966 Convection at high Rayleigh number. In Proc. 11th Intl Cong. Applied Mech. (ed. H. Gortler), p. 1109. Springer.

Husar, R. B. \& Sparrow, E. M. 1968 Patterns of free convection flow adjacent to horizontal heated surfaces. Intl J. Heat Mass Transfer 11, 1208-1211.

JimÉNEZ, J. \& MoIn, P. 1991 The minimal flow unit in near-wall turbulence. J. Fluid Mech. 225, 213-240.

Kallol Bera 1993 Effect of finite conductivity on free convection. ME thesis, Dept. Mechanical Engng, Indian Institute of Science, Bangalore, India.

Kerr, R. M. 1996 Rayleigh number scaling in numerical convection. J. Fluid Mech. 310, 139-179.

Kitamura, K. \& Kimura, F. 1995 Heat transfer and fluid flow of natural convection adjacent to upward-facing horizontal plates. Intl J. Heat Mass Transfer 38, 3149-3159.

Kraichnan, R. J. 1962 Mixing-length analysis of turbulent thermal convection at arbitrary Prandtl number. Phys. Fluids 5, 1374-1389.

LANDAHL, M. T. 1990 On sublayer streaks. J. Fluid Mech. 212, 593-614.

Lumley, J. L. 1967 The structure of inhomogeneous turbulent flows. In Atmospheric Turbulence and Radio Wave Propagation (ed. A. M. Yaglom \& V. I. Tatarski), pp. 166-178. Moscow: Nauka.

Lundgren, T. S. 1982 Strained spiral vortex model for turbulent fine structure. Phys. Fluids 25, 2193.

Malkus, W. V. R. 1954 The heat transfer and spectrum of thermal turbulence. Proc. R. Soc. Lond. A 225, 195-212.

Patankar, S. V. 1980 Numerical Heat Transfer and Fluid Flow. Hemisphere.

Pera, L. \& Gebhart, B. 1973 a Natural convection boundary layer flow over horizontal and slightly inclined surfaces. Intl J. Heat Mass Transfer 16, 1131-1146.

Pera, L. \& Gebhart, B. $1973 b$ On the stability of natural convection boundary layer flow over horizontal and slightly inclined surfaces. Intl J. Heat Mass Transfer 16, 1147-1163.

Prasad, A. K. \& Gonuguntla, P. V. 1996 Turbulence measurements in nonpenetrative thermal convection. Phys. Fluids 8, 2460-2470.

Pullin, D. I. \& Saffman, P. G. 1993 On the Lundgren-Townsend model of turbulent fine scales. Phys. Fluids A 5, 126-145.

Rotem, Z. \& Classen, L. 1969 Natural convection above unconfined horizontal surfaces. J. Fluid Mech. 39, 173-192.

SANGHI, S. \& Aubry, N. 1993 Mode interaction models for near-wall turbulence. J. Fluid Mech. 247, 455-488.

SigGia, E. D. 1994 High Rayleigh number convection. Ann. Rev. Fluid Mech. 26, 137-168.

Somerscales, E. F. C. \& GazdA, I. W. 1969 Thermal convection in high Prandtl number liquids at high Rayleigh numbers. Intl J. Heat Mass Transfer 12, 1491-1511.

Spangenberg, W. H. \& Rowland, W. R. 1961 Convective circulation in water induced by evaporating cooling. Phys. Fluids 4, 743-750. 
Sparrow, E. M., Husar, R. B. \& Goldstein, R. J. 1970 Observations and other characteristics of thermals. J. Fluid Mech. 41, 793-800.

Tamai, N. \& Asaeda, T. 1984 Sheet like plumes near a heated bottom plate at large Rayleigh number. J. Geophys. Res. 89, 727-734.

TANAKA, H. \& MiYatA, H. 1980 Turbulent natural convection in a horizontal water layer heated from below. Intl J. Heat Mass Transfer 23, 1273-1281.

Theerthan, S. A. \& Arakeri, J. H. 1994 Planform structure of turbulent Rayleigh-Bénard convection. Intl Commun. Heat Mass Transfer 21, 561-572.

Tilgner, A., Belmonte, A. \& Libchaber, A. 1993 Temperature and velocity profiles of turbulent convection in water. Phys. Rev. E 47, 2253-2256.

Townsend, A. A. 1951 On the fine-scale structure of turbulence. Proc. R. Soc. Lond. A 208, 534.

Townsend, A. A. 1959 Temperature fluctuations over a heated horizontal surface. J. Fluid Mech. 5, 209-241.

Turner, J. S. 1973 Buoyancy Effects in Fluids, p. 226. Cambridge University Press

Van Doormane, J. P. \& Raithby, G. D. 1984 Enhancements of the SIMPLE method for predicting incompressible fluid flows. Numer. Heat Transfer 7, 147-163.

Wu, X.-Z. \& LibChaber, A. 1992 Scaling relations in thermal turbulence: The aspect-ratio dependence. Phys. Rev. A 45, 843-845.

Zocchi, G., Moses, E. \& Libchaber, A. 1990 Coherent structures in turbulent convection, an experimental study. Physica A 166, 387-407. 